\title{
Fock 空间及其相关算子
}

献给史济怀教授 80 华诞

\author{
胡璋剑*，吕小芬 \\ 湖州师范学院数学系, 湖州 313000 \\ E-mail: huzj@hutc.zj.cn, lvxf@hutc.zj.cn
}

收稿日期: 2015-03-27; 接受日期: 2015-06-04; * 通信作者

国家自然科学基金 (批准号: 11271124, 11171277 和 11426104) 和浙江省自然科学基金 (批准号: LY15A010014 和 LQ13A010005) 资助项目

摘要 本文是关于 Fock 空间及其上相关算子研究的综述, 包括 Fock 空间的定义、Fock 空间的 Bergman 核估计和 Carleson 测度及其范数等价刻画等基本内容, 并且展示了对 Fock 空间上相关线性算子 (如 复合算子、广义 Cesàro 算子、Toeplitz 算子和 Hankel 算子及某些线性算子所生成的闭代数) 若干特 性研究的最新进展, 这些特性包括有界性、紧性、Schatten 类和 Schatten-Herz 类等.

关键词 Fock 空间 Bergman 核 Carleson 测度 Toeplitz 算子 Hankel 算子

MSC (2010) 主题分类 $47 \mathrm{~B} 38$

\section{1 引言}

有界区域上全纯函数空间理论在最近的几十年有着极大的发展, 这些空间涵盖单位圆盘 (或单位 球、典型域、强拟凸域) 上的 Hardy 空间、Bergman 空间、BMOA 空间和 Bloch 空间等. 对这些空间的 研究可参见专著 [1-3] 等. 相较于有界区域, 对无界区域上全纯函数空间的研究要少得多. 这可以从关 于全纯函数空间的专著大多集中于有界区域的研究而得到印证. Fock 空间是定义在无界域上的全纯 函数空间. 有关 Fock 空间的研究也已有几十年的历史, 可追溯到 20 世纪 60 年代. 最早引入 Fock 空 间的是 Bargmann (虽然那时对这个空间还没有这样的称呼), 参见文献 [4]. 学者们发现 Fock 空间与 量子理论有着天然的联系. 例如, 与谐振子的量子理论相关联, 稠定义的微分算子 $D: f \mapsto f^{\prime}$ 被称为 湮灭算子 (the annihilation operator), 其形式对偶为乘法算子 $D^{*}: f \mapsto z f, D^{*}$ 也被称为产生算子 (the creation operator). 在文献 [4] 之后, Fock 空间引起了学者们更多的关注, 近年更是吸引了众多学者的 研究兴趣, 可参见文献 [5-11] 等, 许多学者也称之为 Segal-Bargmann 空间, 参见文献 [12-15]. 2012 年, 有关 Fock 空间的第一本专著由 Springer 出版社出版, 参见文献 [16].

本文的目的是对 Fock 空间及其相关算子理论的最新研究进展作介绍. 包括如下内容: 各类 Fock 空间的定义、关于 Bergman 核函数的基本估计、Fock 空间范数的等价刻画、Fock-Carleson 测度、复 合算子、广义 Cesaro 算子、Toeplitz 算子、Hankel 算子、弱局部 Toeplitz 算子代数. 
在展开详细介绍之前, 我们先约定若干记号. 我们以 $\mathbb{C}^{n}$ 记 $n$ 维复 Euclid 空间, 以 $d v$ 记 $\mathbb{C}^{n}$ 上规 范化的 Lebesgue 体积测度. 经典 Lebesgue 空间 $L^{p}$ 表示 $\mathbb{C}^{n}$ 上满足

$$
\|f\|_{L^{p}}=\left(\int_{\mathbb{C}^{n}}|f|^{p} d v\right)^{\frac{1}{p}}<\infty
$$

的 Lebesgue 可测函数 $f$ 的全体. 对 $z, w \in \mathbb{C}^{n},\langle z, w\rangle=\sum_{k=1}^{n} z_{k} \overline{w_{k}},|z|=\langle z, z\rangle^{\frac{1}{2}}$. 给定区域 $\Omega \subseteq \mathbb{C}^{n}$, 以 $H(\Omega)$ 表示 $\Omega$ 上全纯函数的全体. 对 $f \in H(\Omega)$ 及 $n$ 重指标 $\beta=\left(\beta_{1}, \beta_{2}, \ldots, \beta_{n}\right)$, 置 $D^{\beta} f=\frac{\partial^{\beta} f}{\partial z^{\beta}}$ 为 $f$ 的偏导数, $|\beta|=\sum_{j=1}^{n} \beta_{j}$. 给定 $z \in \mathbb{C}^{n}$ 及 $r>0$, 记 $B(z, r)=\left\{w \in \mathbb{C}^{n}:|w-z|<r\right\}$, $B(z, r)^{c}=\mathbb{C}^{n} \backslash B(z, r) ;$ 在 $n=1$ 时, 又记 $D(z, r)=\{w \in \mathbb{C}:|w-z|<r\}$.

文中, 符号 $C$ 表示与所讨论的量无关的正常数, 每次出现未必相同. 对给定的两个量 $A$ 和 $B$ (可 以是 $(1,1)$ - 型微分形式, 也可以是区域上的函数或函数空间上的泛函), 我们称 $A$ 和 $B$ 是等价的 (用 记号 $A \simeq B$ 表示) 是指存在正常数 $C$ 使得 $C^{-1} B \leqslant A \leqslant C B$.

\section{Fock 空间的定义}

给定 $0<p<\infty$, 经典的 Fock 空间是指 $\mathbb{C}$ 上满足

$$
\left(\int_{\mathbb{C}}\left|f(z) \mathrm{e}^{-\frac{1}{2}|z|^{2}}\right|^{p} d v(z)\right)^{\frac{1}{p}}<\infty
$$

的单变量整函数 $f$ 所组成的集合. 稍作一般化, 给定 $\alpha>0$, Fock 空间 $F_{\alpha}^{p}$ 定义为

$$
F_{\alpha}^{p}=\left\{f \in H\left(\mathbb{C}^{n}\right):\left\{\int_{\mathbb{C}^{n}}\left|f(z) \mathrm{e}^{-\frac{\alpha}{2}|z|^{2}}\right|^{p} d v(z)\right\}^{\frac{1}{p}}<\infty\right\} .
$$

在过去的几十年中, 学者们对 Fock 空间的研究主要是针对 $F_{\alpha}^{p}$ (而且是在 $n=1$ 情形) 展开的, 可参见 文献 $[4-7,12,13,16]$ 等. 2012 年, Cho 和 Zhu ${ }^{[17]}$ 引进了 Fock-Sobolev 空间 $F^{p, m}$ 的概念, 它们是 $F_{1}^{p}$ 的推广. 按照文献 [17], 给定 $0<p<\infty$ 及非负整数 $m$, 定义 Fock-Sobolev 空间 $F^{p, m}$ 为

$$
F^{p, m}=\left\{f \in H\left(\mathbb{C}^{n}\right):\|f\|_{F^{p, m}}=\sum_{|\beta| \leqslant m}\left\{\int_{\mathbb{C}^{n}}\left|D^{\beta} f(z) \mathrm{e}^{-\frac{1}{2}|z|^{2}}\right|{ }^{p} d v(z)\right\}^{\frac{1}{p}}<\infty\right\} .
$$

随后, 文献 [18-23] 对 Fock-Sobolev 空间进行了研究. 同样是在 2012 年, Schuster 和 Varolin ${ }^{[24]}$ 定义 了一类由某种权函数 $\varphi$ 所诱导的 Fock 空间 $F_{\varphi}^{p}$. 具体地, 记微分算子 $d=\bar{\partial}+\partial, d^{c}=\frac{\sqrt{-1}}{4}(\bar{\partial}-\partial)$, $\omega_{0}=d d^{c}|z|^{2}=\frac{i}{2} \sum_{k=1}^{n} d \overline{z_{k}} \wedge d z_{k}$ 是 $\mathbb{C}^{n}$ 上的 Kähler 形式. 实值函数 $\varphi \in C^{2}\left(\mathbb{C}^{n}\right)$, 在流的意义下 (in the sense of current) 满足 $d d^{c} \varphi \simeq \omega_{0}$. 对 $0<p<\infty$, 以 $L_{\varphi}^{p}$ 表示 $\mathbb{C}^{n}$ 上满足

$$
\|f\|_{p, \varphi}=\left(\int_{\mathbb{C}^{n}}\left|f(z) \mathrm{e}^{-\varphi(z)}\right|^{p} d v(z)\right)^{\frac{1}{p}}<\infty
$$

的 Lebesgue 可测函数的全体. Fock 空间 $F_{\varphi}^{p}$ 定义为

$$
F_{\varphi}^{p}=L_{\varphi}^{p} \cap H\left(\mathbb{C}^{n}\right), \quad 0<p<\infty,
$$

及

$$
F_{\varphi}^{\infty}=\left\{f \in H\left(\mathbb{C}^{n}\right):\|f\|_{\infty, \varphi}=\sup _{z \in \mathbb{C}^{n}}|f(z)| \mathrm{e}^{-\varphi(z)}<\infty\right\}
$$


显而易见, 在 $n=1$ 和 $\varphi(z)=\frac{1}{2}|z|^{2}$ 时, $F_{\varphi}^{p}$ 就是经典的 Fock 空间. 而对给定的 $f \in H\left(\mathbb{C}^{n}\right)$, Cho 和 $\mathrm{Zhu}{ }^{[17]}$ 证明了 $f \in F^{p, m}$ 的充要条件是

$$
\left(\left.\left.\int_{\mathbb{C}^{n}}|| z\right|^{m} f(z) \mathrm{e}^{-\frac{1}{2}|z|^{2}}\right|^{p} d v(z)\right)^{\frac{1}{p}}<\infty
$$

并且上式左端与 $F^{p, m}$ 范数等价. 这说明 $F^{p, m}$ 可以通过类似于 $(2.1)$ 来定义, 只要取 $\varphi(z)=\frac{1}{2}|z|^{2}$ $-m \log |z|$. 但 $\frac{1}{2}|z|^{2}-m \log |z|$ 并不是多重次调和的, 不满足 Schuster 和 Varolin 在文献 [24] 中对权函 数的要求. 另一方面, 通过范数的等价刻画容易看出, 取定适当大的 $A>0, \varphi(z)=\frac{1}{2}|z|^{2}-\frac{m}{2} \ln \left(A+|z|^{2}\right)$ 满足 $\varphi \in C^{2}\left(\mathbb{C}^{n}\right)$ 及 $d d^{c} \varphi \simeq \omega_{0}$, 并且由这样的 $\varphi$ 所定义的 $F_{\varphi}^{p}$ 就是 $F^{p, m}$. 所以, Fock-Sobolev 空间也 是 $F_{\varphi}^{p}$ 的某种特殊情形.

由文献 $\left[24\right.$, 命题 2.3] 可知, 存在常数 $C>0$ 使得对所有 $f \in H\left(\mathbb{C}^{n}\right)$ 成立

$$
\left|f(z) \mathrm{e}^{-\varphi(z)}\right|^{p} \leqslant C r^{-2 n} \int_{B(z, r)}\left|f(w) \mathrm{e}^{-\varphi(w)}\right|^{p} d v(w) .
$$

利用上式, 不难得到

$$
F_{\varphi}^{p} \subset F_{\varphi}^{q}, \quad 0<p<q \leqslant \infty .
$$

限制在 $n=1$ 情形, 学者们还引入过一类更为一般的加权 Fock 空间 $F_{\phi}^{p}$. 一般地, 我们称 $\mathbb{C}$ 上的 一个正 Borel 测度 $\nu$ 满足双倍条件 (doubling condition) 是指, 存在正常数 $C$ 使得对任意的 $z \in \mathbb{C}$ 及 $r>0$ 成立

$$
\nu(D(z, 2 r)) \leqslant C \nu(D(z, r)) .
$$

给定 $\mathbb{C}$ 上不处处为零的次调和函数 $\phi, \Delta \phi d v$ 满足双倍条件, 用 $\phi$ 代替 (2.1) 中的 $\varphi$ 来定义相应的 Lebesgue 空间 $L_{\phi}^{p}$, 再定义加权 Fock 空间 $F_{\phi}^{p}=L_{\phi}^{p} \cap H(\mathbb{C})$. 对这类加权 Fock 空间的研究开始于 20 世纪 90 年代, 参见文献 [25]. 之后有诸多学者致力于 $F_{\phi}^{p}$ 的研究, 可参见文献 [26-30] 等. 注意到, 在 $n=1$ 时满足 $d d^{c} \varphi \simeq \omega_{0}$ 的权函数 $\varphi$ 的 Laplace 必满足双倍条件, 所以, $\mathbb{C}$ 上的 Fock 空间 $F_{\varphi}^{p}$ 是加权 Fock 空间 $F_{\phi}^{p}$ 的特殊情形. 对 $m>1$, 函数 $\phi(z)=|z|^{m}$ 的 Laplace 满足双倍条件. 而由 $\phi(z)=|z|^{2 m}$ ( $m$ 为大于 1 的整数) 所定义的 $F_{\phi}^{2}$ 就是文献 $[31,32]$ 所讨论的 Fock 空间.

此外, 学者们还研究了由其他权函数所定义的 Fock 空间. 例如, Seip 和 Youssfi ${ }^{[33]}$ 定义了加权 Fock 空间 $F_{\psi}^{2}$, 这里的权函数 $\psi$ 是径向函数, $\psi:[0, \infty) \rightarrow[0, \infty) \in C^{3}$, 满足条件 $\psi(x)>0, \psi^{\prime}(x) \geqslant 0$, $\psi^{\prime \prime}(x) \geqslant 0$. Constantin 和 Peláez ${ }^{[34]}$ 在 2015 年又定义了一类新的加权 Fock 空间 $F_{\psi}^{p}$, 其中 $\psi$ 也是径 向函数, $\psi:[0, \infty) \rightarrow(0, \infty) \in C^{2}$, 且存在 $\mathbb{C}$ 上的一个径向可微正值函数 $\tau, \tau^{\prime} \leqslant 0$,

$$
\lim _{r \rightarrow \infty} \tau(r)=0, \quad \lim _{r \rightarrow \infty} \tau^{\prime}(r)=0
$$

使得

$$
\tau(z) \simeq \begin{cases}1, & 0 \leqslant|z|<1 \\ (\Delta \psi(|z|))^{-\frac{1}{2}}, & |z| \geqslant 1 .\end{cases}
$$

值得注意的是, 对加权 Fock 空间 $F_{\phi}^{p}$ 而言, 与 (2.2) 相类似的估计仍然成立, 但 (2.3) 一般不再成 立. 事实上, 只要取 $\phi(z)=|z|^{4}$, 对所有 $p, q>0, p \neq q$, 由文献 [34] 知, $F_{\phi}^{p} \backslash F_{\phi}^{q} \neq \emptyset$ 且 $F_{\phi}^{q} \backslash F_{\phi}^{p} \neq \emptyset$. 


\section{Bergman 核函数}

易知, 当 $1 \leqslant p \leqslant \infty$ 时, 在范数 $\|\cdot\|_{p, \varphi}$ 下, $F_{\varphi}^{p}$ 是 Banach 空间; 当 $0<p<1$ 时, 在度量 $d(f, g)$ $=\|f-g\|_{p, \varphi}^{p}$ 下, $F_{\varphi}^{p}$ 是 Fréchet 空间. 特别地, 在内积

$$
\langle f, g\rangle_{\varphi}=\int_{\mathbb{C}^{n}} f \bar{g} \mathrm{e}^{-2 \varphi} d v
$$

下, $F_{\varphi}^{2}$ 成为一个 Hilbert 空间. 当 $\varphi(z)=\frac{\alpha}{2}|z|^{2}$ 时, 我们把 $\langle\cdot, \cdot\rangle_{\varphi}$ 简记成 $\langle\cdot, \cdot\rangle_{\alpha}$. 由估计式 $(2.2)$ 可以看 出, 点值泛函 $\Lambda_{z}: f \mapsto f(z)$ 是 $F_{\varphi}^{p}$ 上的有界线性泛函. 由此可知, 存在 $F_{\varphi}^{2}$ 上的再生核 (reproducing kernel) $K_{\varphi}(\cdot, \cdot) . K_{\varphi}(\cdot, \cdot)$ 也称为 $F_{\varphi}^{2}$ 上的 Bergman 核. 当 $\varphi(z)=\frac{\alpha}{2}|z|^{2}$ 时, 直接计算可得 Bergman 核 函数 $K_{\alpha}(z, w)=\mathrm{e}^{\alpha\langle z, w\rangle}$, 可参见文献 [8]. 对 Fock-Sobolev 空间 $F^{p, m}$, 关于 Bergman 核函数表达式可 参见文献 [17]. 在文献 [24] 中, Schuster 和 Varolin 利用 $\bar{\partial}$ 问题的 $L^{2}$ 估计, 得到了 $K_{\varphi}(\cdot, \cdot)$ 的若干估 计, 这些估计对 $F_{\varphi}^{p}$ 上函数空间和算子理论的研究是十分重要的.

定理 3.1 (A) 存在常数 $C, \theta>0$ 使得对 $z, w \in \mathbb{C}^{n}$ 成立

$$
\left|K_{\varphi}(z, w)\right| \leqslant C \mathrm{e}^{\varphi(z)+\varphi(w)-\theta|z-w|} ;
$$

(B) 存在常数 $r_{0}>0$ 使得对任意 $z \in \mathbb{C}^{n}$ 和 $w \in B\left(z, r_{0}\right)$ 总有

$$
\left|K_{\varphi}(z, w)\right| \simeq \mathrm{e}^{\varphi(z)+\varphi(w)}
$$

(C) 对所有 $0<p \leqslant \infty$ 有

$$
\left\|K_{\varphi}(\cdot, z)\right\|_{p, \varphi} \simeq \mathrm{e}^{\varphi(z)} \simeq \sqrt{K(z, z)}, \quad z \in \mathbb{C}^{n} .
$$

关于加权 Fock 空间 $F_{\phi}^{2}$ 上 Bergman 核的相应估计已经由文献 $[28,30]$ 给出. 给定 $\mathbb{C}$ 上不处处为 零的次调和函数 $\phi, \nu=\Delta \phi d v$ 满足双倍条件, 对 $z \in \mathbb{C}$, 记 $\rho(z)$ 是使得 $\nu(D(z, \rho(z)))=1$ 的圆盘半径. 置 $D^{r}(z)=D(z, r \rho(z))$.

定理 3.2 (A) 存在常数 $C, \epsilon>0$ 使得对 $z, w \in \mathbb{C}^{n}$ 成立

$$
|K(w, z)| \leqslant C \frac{\mathrm{e}^{\phi(w)+\phi(z)}}{\rho(w) \rho(z)} \mathrm{e}^{-\left(\frac{|z-w|}{\rho(z)}\right)^{\epsilon}} ;
$$

(B) 存在常数 $r_{0}>0$ 使得对任意 $z \in \mathbb{C}$ 和 $w \in D^{r_{0}}(z)$ 总有

$$
|K(w, z)| \simeq \frac{\mathrm{e}^{\phi(w)+\phi(z)}}{\rho(w) \rho(z)} \simeq \frac{\mathrm{e}^{\phi(w)+\phi(z)}}{\rho(z)^{2}} ;
$$

(C) 对所有 $0<p \leqslant \infty$ 有

$$
\|K(\cdot, z)\|_{p, \phi} \simeq \mathrm{e}^{\phi(z)} \rho(z)^{\frac{2}{p}-2}, \quad z \in \mathbb{C} .
$$

利用 Bergman 核, 就可以写出 Bergman 投影 $P$,

$$
P f(\cdot)=\int_{\mathbb{C}^{n}} f(w) K_{\varphi}(\cdot, w) \mathrm{e}^{-2 \varphi(w)} d v(w) .
$$


由 $P$ 的定义, 对幂次为 2 的 Fock 空间中的函数 $f$, 恒有 $P f=f$. 利用定理 3.1 (或定理 3.2), 由直 接的运算可知, 算子 $P$ 在 $L_{\varphi}^{\infty}$ 及 $L_{\varphi}^{1}$ 上分别是有界的. 由 Marcinkiewicz 插值定律, $P$ 在 $L_{\varphi}^{p}$ 上有界, $1 \leqslant p \leqslant \infty$. 由此容易证明 $P$ 是 $F_{\varphi}^{p}$ 上的恒等映射. 再由 $(2.3)$, 我们就得

$$
P f=f
$$

关于一切 $0<p \leqslant \infty$ 和 $f \in F_{\varphi}^{p}$ 成立. 对 $1 \leqslant p \leqslant \infty$, (3.1) 对 $f \in F_{\phi}^{p}$ 也成立. 而对 $p \in(0,1)$ 时的 $p$ 次 Fock 空间, Bergman 投影在其上的再生性依赖于定义这类空间的权函数的特性.

\section{4 范数等价刻画}

对有界区域而言, 从 Hardy 和 Littlewood 时代开始, 人们就知道 Hardy 空间 $H^{2}$ 中的函数可以 用它们导数的某种积分来等价刻画. 用函数的导数、高阶导数来等价刻画 Bergman 空间、Bloch 空 间、BMOA 空间、Besov 空间等, 都是熟知的结果. 这些等价刻画对函数空间上算子理论的研究提供 了很多的便利. 例如, 文献 [35] 利用 “混合模空间 (Bergman 空间)” 的导数刻画建立了广义 Cesaro 算子的有界性和紧性. 关于 Fock 空间, 人们曾一度认为用导数来对其进行刻画是不可能的. Furdui [5] 就曾说 “不同于 Bergman 空间, Fock 空间无法用偏导数的 $L^{p}$ 范数来刻画”. 但事实上, 文献 [36] 于 2013 年就 $\varphi(z)=\frac{\alpha}{2}|z|^{2}$ 所定义的 $\mathbb{C}^{n}$ 上 Fock 空间, 利用 $m$ 阶偏导数 (或 $m$ 阶径向导数) 得到了 $F_{\alpha}^{p}$ 的范数等价式. 也是在 2013 年, 对经典的一元 Fock 空间 $F_{1}^{p}$, Constantin ${ }^{[37]}$ 得到了同样的结果. 下述 定理 4.1 摘自文献 [36], 当 $n=1, \alpha=1$ 时, 这就是文献 [37, 命题 1].

定理 4.1 设 $0<p, \alpha<\infty$. 对 $f \in H\left(\mathbb{C}^{n}\right)$, 下述各量互相等价:

(A) $\|f\|_{p, \alpha}$;

(B) $\sum_{|\beta| \leqslant m-1}\left|D^{\beta} f(0)\right|+\left\{\sum_{|\beta|=m} \int_{\mathbb{C}^{n}}\left|D^{\beta} f(z) \frac{1}{(1+|z|)^{m}} \mathrm{e}^{-\frac{\alpha}{2}|z|^{2}}\right|^{p} d v(z)\right\}^{\frac{1}{p}}$

(C) $|f(0)|+\left\{\int_{\mathbb{C}^{n}}\left|\Re^{m} f(z) \frac{1}{(1+|z|)^{2 m}} \mathrm{e}^{-\frac{\alpha}{2}|z|^{2}}\right|^{p} d v(z)\right\}^{\frac{1}{p}}$, 其中 $\Re f(z)=\sum_{j=1}^{\infty} z_{j} \frac{\partial f}{\partial z_{j}}(z)$ 表示 $f$ 的径向导 数, $\Re^{m} f=\Re\left(\Re^{m-1} f\right)$.

2015 年, Constantin 和 Peláez [34] 对满足第 2 节所述条件的权函数 $\psi$ 所定义的一元函数空间 $F_{\psi}^{p}$, 给出了如下的定理 4.2. 当 $\psi(z)=\frac{\alpha}{2}|z|^{2}$ 时, 定理 4.2 和 $n=m=1$ 情形的定理 4.1 是一致的.

定理 4.2 设 $0<p<\infty, f \in H(\mathbb{C})$, 则

$$
\|f\|_{p, \psi}^{p} \simeq|f(0)|^{p}+\int_{\mathbb{C}}\left|f^{\prime}(z)\right|^{p} \frac{\mathrm{e}^{-p \psi(z)}}{\left(1+\psi^{\prime}(z)\right)^{p}} d v(z) .
$$

\section{Carleson 测度}

Carleson 测度在函数空间理论的研究中起着十分重要的作用. 在 Hardy 空间理论中, Carleson 测 度在 $H^{1}$-BMO 对偶、Corona 问题、Toeplitz 算子及广义 Cesàro 算子等问题的讨论中都扮演着关键角 色. 在 Bergman 空间情形, Carleson 测度的类似作用也可举出很多. 可见, Carleson 测度在 Fock 空间 及其上算子的研究中也有相应的地位.

在展开对 Carleson 测度的叙述之前, 我们先给出一些常用的记号. 给定 $\mathbb{C}^{n}$ 上的 Borel 测度 $\mu, \mu$ 的平均函数和 $t$ - 次 Berezin 变换 $(t>0)$ 分别定义为

$$
\widehat{\mu}_{r}(z)=\frac{\mu(B(z, r))}{v(B(z, r))}, \quad z \in \mathbb{C}^{n}
$$


和

$$
\widetilde{\mu}_{t}(z)=\int_{\mathbb{C}^{n}}\left|k_{\varphi, z}(w)\right|^{t} \mathrm{e}^{-t \varphi(w)} d \mu(w), \quad z \in \mathbb{C}^{n},
$$

其中 $k_{\varphi, z}(w)=\frac{K_{\varphi}(w, z)}{\sqrt{K_{\varphi}(z, z)}}$. 当 $\varphi(z)=\frac{\alpha}{2}|z|^{2}$ 时, $k_{\varphi, z}$ 简记为 $k_{\alpha, z}$. 注意到, $\tilde{\mu}_{2}$ 就是经典的 Berezin 变换, 简记为 $\widetilde{\mu}$. 给定 $\mathbb{C}^{n}$ 上的可测函数 $f$ 及 $d \mu=f d v$, 简记 $\widehat{f}_{r}=\widehat{\mu}_{r}, \widetilde{f}_{t}=\widetilde{\mu}_{t}$.

对 $r>0$ 和 $\mathbb{C}^{n}$ 中的序列 $\left\{a_{k}\right\}_{k=1}^{\infty}$, 如果 $\left\{B\left(a_{k}, r\right)\right\}_{k=1}^{\infty}$ 覆盖 $\mathbb{C}^{n}$ 且 $\left\{B\left(a_{k}, \frac{r}{2}\right)\right\}_{k=1}^{\infty}$ 彼此互不相交, 则称 $\left\{a_{k}\right\}_{k=1}^{\infty}$ 是 $\mathbb{C}^{n}$ 上的 $r$ - 格.

给定 $\mathbb{C}^{n}$ 上的正 Borel 测度 $\mu$ (简记为 $\mu \geqslant 0$ ), $0<p<\infty$, 空间 $L^{p}(\varphi, \mu)$ 是 $\mathbb{C}^{n}$ 上满足

$$
\|f\|_{L^{p}(\varphi, \mu)}=\left(\int_{\mathbb{C}^{n}}|f(z)|^{p} \mathrm{e}^{-p \varphi(z)} d \mu(z)\right)^{\frac{1}{p}}<\infty
$$

的 $\mu$ - 可测函数 $f$ 的全体.

定义 5.1 设 $\mu \geqslant 0,0<p, q<\infty$. 如果嵌入算子 $i: F_{\varphi}^{p} \rightarrow L^{q}(\varphi, \mu)$ 有界, 即存在正常数 $C$, 使得 对任意 $f \in F_{\varphi}^{p}$, 有

$$
\left(\int_{\mathbb{C}^{n}}\left|f(z) \mathrm{e}^{-\varphi(z)}\right|^{q} d \mu(z)\right)^{\frac{1}{q}} \leqslant C\|f\|_{p, \varphi},
$$

则称 $\mu$ 是 $(p, q)$-Carleson 测度. 进一步, 如果对任意在 $\mathbb{C}^{n}$ 上内闭一致收玫到 0 的范数有界函数列 $\left\{f_{j}\right\}_{j=1}^{\infty} \subset F_{\varphi}^{p}$, 都有

$$
\lim _{j \rightarrow \infty} \int_{\mathbb{C}^{n}}\left|f_{j}(z) \mathrm{e}^{-\varphi(z)}\right|^{q} d \mu(z)=0,
$$

则称 $\mu$ 是消没 $(p, q)$-Carleson 测度.

对经典的 Fock 空间, Carleson 测度最早由 Isralowitz 和 Zhu 在文献 [6] 中引入. 2012 年, Cho 和 Zhu 在文献 [17] 提出了 Fock-Sobolev 空间的 Carleson 测度. 在文献 [24] 中, 就满足 $d d^{c} \varphi \simeq \omega_{0}$ 的权函 数 $\varphi$ 所定义的 Fock 空间 $F_{\varphi}^{p}(1<p<\infty)$, Schuster 和 Varolin 研究了其上的 $(p, p)$-Carleson 测度. 我 们在文献 [38] 中刻画了 $F_{\varphi}^{p}$ 上的 $(p, q)$-Carleson 测度, 其中 $0<p, q<\infty$, 得到如下结果.

定理 5.2 设 $\mu \geqslant 0,0<p \leqslant q<\infty$, 则下列各款互相等价:

(A) $\mu$ 是 $(p, q)$-Carleson 测度;

(B) 存在 (或对任意) $t>0, \widetilde{\mu}_{t}$ 在 $\mathbb{C}^{n}$ 上有界;

(C) 存在 (或对任意) $\delta>0, \widehat{\mu}_{\delta}$ 在 $\mathbb{C}^{n}$ 上有界;

(D) 存在 (或对任意) $\mathbb{C}^{n}$ 上的 $r$ - 格 $\left\{a_{k}\right\}_{k}$, 序列 $\left\{\widehat{\mu}_{r}\left(a_{k}\right)\right\}_{k}$ 有界. 进一步,

$$
\|i\|_{F_{\varphi}^{p} \rightarrow L^{q}(\varphi, \mu)}^{q} \simeq \sup _{z \in \mathbb{C}^{n}} \widetilde{\mu}_{t}(z) \simeq \sup _{z \in \mathbb{C}^{n}} \widehat{\mu}_{\delta}(z) \simeq \sup _{k} \widehat{\mu}_{r}\left(a_{k}\right) .
$$

定理 5.3 设 $\mu \geqslant 0,0<p \leqslant q<\infty$, 则下列各款互相等价:

(A) $\mu$ 是消没 $(p, q)$-Carleson 测度;

(B) 存在 (或对任意) $t>0$, 当 $z \rightarrow \infty$ 时, $\widetilde{\mu}_{t}(z) \rightarrow 0$;

(C) 存在 (或对任意) $\delta>0$, 当 $z \rightarrow \infty$ 时, $\widehat{\mu}_{\delta}(z) \rightarrow 0$;

(D) 存在 (或对任意) $\mathbb{C}^{n}$ 上的 $r$ - 格 $\left\{a_{k}\right\}_{k=1}^{\infty}$, 当 $k \rightarrow \infty$ 时, $\widehat{\mu}_{r}\left(a_{k}\right) \rightarrow 0$.

定理 5.4 设 $\mu \geqslant 0,0<q<p<\infty$, 则下列各款互相等价:

(A) $\mu$ 是 $(p, q)$-Carleson 测度; 
(B) $\mu$ 是消没 $(p, q)$-Carleson 测度;

(C) 存在 (或对任意) $t>0, \widetilde{\mu}_{t} \in L^{\frac{p}{p-q}}$;

(D) 存在 (或对任意) $\delta>0, \widehat{\mu}_{\delta} \in L^{\frac{p}{p-q}}$;

(E) 存在 (或对任意) $\mathbb{C}^{n}$ 上的 $r$ - 格 $\left\{a_{k}\right\}_{k=1}^{\infty},\left\{\widehat{\mu}_{r}\left(a_{k}\right)\right\}_{k=1}^{\infty} \in l^{\frac{p}{p-q}}$. 进一步,

$$
\|i\|_{F_{\varphi}^{p} \rightarrow L^{q}(\varphi, \mu)}^{q} \simeq\left\|\widetilde{\mu}_{t}\right\|_{L^{\frac{p}{p-q}}} \simeq\left\|\widehat{\mu}_{\delta}\right\|_{L^{\frac{p}{p-q}}} \simeq\left\|\left\{\widehat{\mu}_{r}\left(a_{k}\right)\right\}_{k}\right\|_{l^{\frac{p}{p-q}}} .
$$

注 5.1 (1) 当 $1 \leqslant p=q<\infty$, 定理 5.2 和 5.3 分别是文献 [24] 的定理 5.1 和 5.2. 当 $\varphi(z)=\frac{\alpha}{2}|z|^{2}$ 时, 我们便能得到文献 $[6,10,17]$ 中有关 Carleson 测度刻画的相应结果.

(2) 在 Bergman 空间情形, $(p, q)$-Carleson 测度与 $p$ 和 $q$ 的精确值无关, 但与 $\frac{p}{q}$ 有关, 参见文 献 [39-41]. 定理 5.2 和 5.3 显示, 就 Fock 空间 $F_{\varphi}^{p}$ 而言, 当 $0<p \leqslant q<\infty$ 时, $(p, q)$-Carleson 测度 与 $\frac{p}{q}$ 也无关. 就是说, 若存在 $s>0$ 使得 $\mu$ 是 $(s, s)$-Carleson 测度, 则对所有 $0<p \leqslant q<\infty, \mu$ 是 $(p, q)$-Carleson 测度. 这种特性表明经典 Fock 空间与 Bergman 空间有较大差异.

(3) 在加权 Fock 空间 $F_{\phi}^{p}$ 情形 (其中 $\phi$ 是满足 $\Delta \phi d v$ 为双倍测度的次调和函数), 我们也研究了 $(p, q)$-Carleson 测度, 具体参见文献 [30]. 结果显示, 定理 5.2 和 5.3 所体现出来的 $(p, q)$-Carleson 测度 与值 $\frac{p}{q}$ 无关的现象只有在权函数 $\phi$ 满足 $\rho(\cdot)$ 等价于常数的情形下才会发生.

\section{6 复合算子}

人们对 Hardy 空间、Bergman 空间上复合算子的研究已超过半个世纪. 熟知, 单位圆盘 Hardy 空 间、Bergman 空间上的复合算子总是有界的; 而高维单位球的 Hardy 空间上的复合算子不总是有界 的. 我们也知道复合算子紧性的刻画要用到消没 Carleson 测度. 对 Fock 空间上复合算子的研究最早 出现于 2003 年, 参见文献 [42]. 文中获得了复合算子 $C_{\Phi}$ 在 Fock 空间 $F_{\frac{1}{2}}^{2}$ 上为有界算子 (或紧算子) 的充要条件, 其中 $C_{\Phi} f=f \circ \Phi, \Phi: \mathbb{C}^{n} \rightarrow \mathbb{C}^{n}$ 为全纯映射. 这一结果显示 Fock 空间上的有界复合算 子只能由满足特定条件的仿射变换诱导, 这与有界区域上的结果很不一致. 2014 年, Cho 等人 ${ }^{[19]}$ 讨论 了复合算子在 Fock-Sobolev 空间上的特性, 得到下述的定理 6.1. 当 $m=0$ 时, 定理 6.1 就是文献 [42] 的结果.

定理 6.1 设 $\varphi: \mathbb{C}^{n} \rightarrow \mathbb{C}^{n}$ 为全纯映射, $m$ 为非负整数, 则

(A) $C_{\Phi}$ 在 $F^{2, m}$ 上为有界算子的充要条件是 $\Phi(z)=A z+B$, 其中 $A$ 是 $n \times n$ 矩阵且 $\|A\|$ $:=\sup _{|\zeta| \leqslant 1}|A \zeta| \leqslant 1, B$ 是 $n \times 1$ 向量. 进一步, 若存在 $\zeta \in \mathbb{C}^{n},|A \zeta|=|\zeta|$, 则 $\langle A \zeta, B\rangle=0$.

(B) $C_{\Phi}$ 在 $F^{2, m}$ 上为紧算子的充要条件是 $\Phi(z)=A z+B$, 其中 $\|A\|<1$.

2010 年, Choe 等人 ${ }^{[43]}$ 对 Fock 空间上两个复合算子的线性组合进行了研究. 2014 年, Cho ${ }^{[19]}$ 讨 论了复合算子的有限线性组合在 Fock-Sobolev 空间上的特性.

定理 6.2 设 $\Phi_{1}, \ldots, \Phi_{N}: \mathbb{C}^{n} \rightarrow \mathbb{C}^{n}$ 均为全纯映射, 对 $j \neq k$ 有 $\Phi_{j} \neq \Phi_{k}, a_{1}, \ldots, a_{N} \in \mathbb{C} \backslash\{0\}, m$ 为非负整数, 则

(A) $a_{1} C_{\Phi_{1}}+\cdots+a_{N} C_{\Phi_{N}}$ 在 $F^{2, m}$ 上为有界算子的充要条件是对任意的 $j=1, \ldots, N, C_{\Phi_{j}}$ 在 $F^{2, m}$ 上为有界算子;

(B) $a_{1} C_{\Phi_{1}}+\cdots+a_{N} C_{\Phi_{N}}$ 在 $F^{2, m}$ 上为紧算子的充要条件是对任意的 $j=1, \ldots, N, C_{\Phi_{j}}$ 在 $F^{2, m}$ 上为紧算子.

当 $m=0, N=2$ 时, 定理 6.2 就是文献 [43] 的主要结果. 


\section{7 广义 Cesàro 算子和复合型积分算子}

有关 Cesàro 算子的研究最早源于三角级数的 Cesàro 求和, 参见文献 [44]. 2003 年, 文献 [35] 首 次引入多复变广义 Cesàro 算子的概念. 在 $H\left(\mathbb{C}^{n}\right)$ 上, 广义 Cesaro 算子也是适定的. 给定 $g \in H\left(\mathbb{C}^{n}\right)$, 在 $H\left(\mathbb{C}^{n}\right)$ 定义广义 Cesaro 算子 $V_{g}$,

$$
V_{g} f(z)=\int_{0}^{1} f(t z) \Re g(t z) \frac{d t}{t}, \quad z \in \mathbb{C}^{n} .
$$

显然, 它是一个线性算子. 我们研究了 $V_{g}$ 在多复变 Fock 空间上的有界性和紧性, 下面的定理 7.1 是 文献 $[36]$ 的主要结果.

定理 7.1 设 $g \in H\left(\mathbb{C}^{n}\right), 0<p \leqslant q<\infty$, 则

(A) $V_{g}: F_{\alpha}^{p} \rightarrow F_{\alpha}^{q}$ 为有界算子的充要条件是 $g$ 为阶数不超过 2 的多项式;

(B) $V_{g}: F_{\alpha}^{p} \rightarrow F_{\alpha}^{q}$ 为紧算子的充要条件是 $g$ 为阶数不超过 1 的多项式.

定理 7.2 设 $g \in H\left(\mathbb{C}^{n}\right), 0<q<p<\infty$, 则下列各款互相等价:

(A) $V_{g}: F_{\alpha}^{p} \rightarrow F_{\alpha}^{q}$ 为有界算子.

(B) $V_{g}: F_{\alpha}^{p} \rightarrow F_{\alpha}^{q}$ 为紧算子.

(C) 当 $\frac{p q}{p-q}>2 n$ 时, $g$ 为阶数不超过 1 的多项式; 当 $\frac{p q}{p-q} \leqslant 2 n$ 时, $g$ 为常数函数.

在单复变情形, Constantin ${ }^{[37]}$ 也研究了算子 $V_{g}$ 在经典 Fock 空间上的算子特性. 当 $n=1, \alpha=1$ 时, 定理 7.1 和 7.2 就是文献 [37] 的定理 1.

Constantin ${ }^{[37]}$ 还研究了广义 Cesàro 算子在 Hilbert 空间 $F_{1}^{2}$ 上的 Schatten 类性质. 在列出文 献 [37] 的相关结果之前, 我们介绍一下 Hilbert 空间 $X$ 上线性算子的 Schatten 类.

定义 7.3 设线性算子 $T$ 是 Hilbert 空间 $X$ 上的紧算子. $T$ 的奇异值 (singular values) 定义为

$$
s_{k}(T)=\inf \{\|T-J\|: \operatorname{rank} J \leqslant k\} .
$$

若 $T$ 的奇异值序列 $\left\{s_{k}(T)\right\}_{k}$ 属于空间 $l^{p}, 0<p<\infty$, 则称 $T$ 属于 Schatten- $p$ 类 $S_{p}(X)$, 记

$$
\|T\|_{S_{p}}=\left(\sum_{k}\left|s_{k}(T)\right|^{p}\right)^{\frac{1}{p}} .
$$

定理 7.4 设 $V_{g}$ 是 $F_{1}^{2}$ 上的紧算子, 则

(A) 对所有 $p>2, V_{g} \in S_{p}\left(F_{1}^{2}\right)$;

(B) $V_{g} \in S_{2}\left(F_{1}^{2}\right)$ 的充要条件是 $g$ 为常数函数.

此外, Constantin 和 Peláez ${ }^{[34]}$ 在 2014 年研究了广义 Cesàro 算子在加权 Fock 空间上的有界性、 紧性和 Schatten 类.

给定 $g, \psi \in H(\mathbb{C})$, 学者们对更一般的复合型积分算子 $V_{(g, \psi)}$ 进行了研究, 其中

$$
V_{(g, \psi)} f(z)=\int_{0}^{z} f \circ \psi(w) g^{\prime}(w) d w, \quad z \in \mathbb{C} .
$$

显然, 当 $\psi(w)=w$ 时, $V_{(g, \psi)}$ 就是广义 Cesàro 算子. Mengestie ${ }^{[45,46]}$ 讨论了不同 Fock 空间之间算 子 $V_{(g, \psi)}$ 的有界性和紧性, 以及 $F_{\alpha}^{2}$ 上 $V_{(g, \psi)}$ 的 Schatten 类特征, 得到如下结果. 这里的 $B_{\psi, \alpha}\left(|g|^{q}\right)$ 定 义为

$$
B_{\psi, \alpha}\left(|g|^{q}\right)(z)=\int_{\mathbb{C}} \frac{\left|\mathrm{e}^{\alpha\langle\psi(w), z\rangle-\frac{\alpha}{2}|z|^{2}} g^{\prime}(w)\right|^{p}}{\left((1+|w|) \mathrm{e}^{\frac{\alpha}{2}|w|^{2}}\right)^{p}} d v(w) .
$$


定理 7.5 设 $0<p \leqslant q \leqslant \infty, \psi, g \in H(\mathbb{C})$, 则

(A) $V_{(g, \psi)}: F_{\alpha}^{p} \rightarrow F_{\alpha}^{q}$ 是有界算子的充要条件是 $B_{\psi, \alpha}\left(|g|^{q}\right)$ 在 $\mathbb{C}$ 上有界. 进一步,

$$
\left\|V_{(g, \psi)}\right\|_{F_{\alpha}^{p} \rightarrow F_{\alpha}^{q}} \simeq \sup _{z \in \mathbb{C}} B_{\psi, \alpha}\left(|g|^{q}\right)(z) .
$$

(B) $V_{(g, \psi)}: F_{\alpha}^{p} \rightarrow F_{\alpha}^{q}$ 是紧算子的充要条件是

$$
\lim _{z \rightarrow \infty} B_{\psi, \alpha}\left(|g|^{q}\right)(z)=0 .
$$

定理 7.6 设 $0<q<p \leqslant \infty, \psi, g \in H(\mathbb{C})$, 则下列各款互相等价:

(A) $V_{(g, \psi)}: F_{\alpha}^{p} \rightarrow F_{\alpha}^{q}$ 是有界算子;

(B) $V_{(g, \psi)}: F_{\alpha}^{p} \rightarrow F_{\alpha}^{q}$ 是紧算子;

(C) $B_{\psi, \alpha}\left(|g|^{q}\right) \in L^{\frac{p}{p-q}}$. 进一步,

$$
\left\|V_{(g, \psi)}\right\|_{F_{\alpha}^{p} \rightarrow F_{\alpha}^{q}} \simeq\left\|B_{\psi, \alpha}\left(|g|^{q}\right)\right\|_{L^{\frac{p}{p-q}}} .
$$

定理 7.7 设 $0<p<\infty, \psi, g \in H(\mathbb{C}), V_{(g, \psi)}$ 是 $F_{\alpha}^{2}$ 上的有界算子, 则 $V_{(g, \psi)} \in S_{p}\left(F_{\alpha}^{2}\right)$ 的充要条 件是 $B_{\psi, \alpha}\left(|g|^{2}\right) \in L^{\frac{p}{2}}$.

\section{Toeplitz 算子}

给定 $0<p<\infty$ 和 $\mathbb{C}^{n}$ 上满足一定条件的 Borel 测度 $\mu$, 由 $\mu$ 所诱导的 Toeplitz 算子 $T_{\mu}$ 在 Fock 空间 $F_{\varphi}^{p}$ 上定义为

$$
T_{\mu} f(\cdot)=\int_{\mathbb{C}^{n}} f(w) K_{\varphi}(\cdot, w) \mathrm{e}^{-2 \varphi(w)} d \mu(w) .
$$

对 $d \mu=g d v, g$ 是可测复值函数, 由 $g d v$ 所诱导的 Toeplitz 算子简记为 $T_{g}$. 当 $\mu \geqslant 0$ 时, 称 $T_{\mu}$ 为正 Toeplitz 算子.

为保证 $T_{\mu}$ 在 Fock 空间 $F_{\varphi}^{2}$ 上可稠定义, 文献 [47] 假设正测度 $\mu$ 满足对所有 $\gamma>0$ 及 $z \in \mathbb{C}^{n}$ 有

$$
\int_{\mathbb{C}^{n}} \mathrm{e}^{-\gamma|z-w|} d \mu(w)<\infty .
$$

类似地, 对文中的 $\mu \geqslant 0$, 我们总假定它诱导的 $T_{\mu}$ 在所讨论的 Fock 空间上可稠定义.

近 30 年来, 有关 Toeplitz 算子在 Fock 空间上的特性已引起学者们的大量关注, 如文献 $[9,14,15]$ 等, 尤以正 Toeplitz 算子被研究得较为彻底, 参见文献 $[6,10,22,24,30,38,47,48] .2010$ 年, Isralowitz 和 Zhu ${ }^{[6]}$ 首次研究了正 Toeplitz 算子在 Fock 空间 $F_{\alpha}^{2}$ 上的有界性、紧性和 Schatten 类. 2011 年, 我 们在文献 [10] 讨论了 $F_{\alpha}^{p}$ 到 $F_{\alpha}^{q}$ 上 $T_{\mu}$ 的有界性和紧性特征. 2014 年, Wang 等人 ${ }^{[22]}$ 讨论了 $T_{\mu}$ 在 Fock-Sobolev 空间 $F^{2, m}$ 上的相应问题. 2012 年, Schuster 和 Varolin ${ }^{[24]}$ 刻画了 $T_{\mu}$ 在 $F_{\varphi}^{p}$ 上为有界 (或紧) 算子的特征, 其中 $1<p<\infty$. 注意到, 这些文献的研究结果都集中在 $p=2$ 或 $1<p<\infty$ 的 情形. 对所有的 $0<p, q \leqslant \infty$, 我们讨论了 $F_{\varphi}^{p}$ 到 $F_{\varphi}^{q}$ 上的正 Toeplitz 算子 $T_{\mu}$ 是有界算子或紧算子的 特征刻画.

定理 8.1 设 $\mu \geqslant 0,0<p \leqslant q \leqslant \infty$, 则下列各款互相等价:

(A) $T_{\mu}: F_{\varphi}^{p} \rightarrow F_{\varphi}^{q}$ 是有界算子;

(B) 存在 (或对任意) $t>0, \widetilde{\mu}_{t}$ 在 $\mathbb{C}^{n}$ 上有界; 
(C) 存在 (或对任意) $\delta>0, \widehat{\mu}_{\delta}$ 在 $\mathbb{C}^{n}$ 上有界;

(D) 存在 (或对任意) $\mathbb{C}^{n}$ 上的 $r$ - 格 $\left\{a_{k}\right\}_{k}$, 序列 $\left\{\widehat{\mu}_{r}\left(a_{k}\right) \rho\left(a_{k}\right)^{2\left(1-\frac{q}{p}\right)}\right\}_{k}$ 有界. 进一步,

$$
\left\|T_{\mu}\right\|_{F_{\varphi}^{p} \rightarrow F_{\varphi}^{q}} \simeq \sup _{z \in \mathbb{C}^{n}} \widetilde{\mu}_{t}(z) \simeq \sup _{z \in \mathbb{C}^{n}} \widehat{\mu}_{\delta}(z) \simeq \sup _{k} \widehat{\mu}_{r}\left(a_{k}\right) .
$$

定理 8.2 设 $\mu \geqslant 0,0<p \leqslant q \leqslant \infty$, 则下列各款互相等价:

(A) $T_{\mu}: F_{\varphi}^{p} \rightarrow F_{\varphi}^{q}$ 是紧算子;

(B) 存在 (或对任意) $t>0$, 当 $z \rightarrow \infty$ 时, $\widetilde{\mu}_{t}(z) \rightarrow 0$;

(C) 存在 (或对任意) $\delta>0$, 当 $z \rightarrow \infty$ 时, $\widehat{\mu}_{\delta}(z) \rightarrow 0$;

(D) 存在 (或对任意) $\mathbb{C}^{n}$ 上的 $r$ - 格 $\left\{a_{k}\right\}_{k}$, 当 $k \rightarrow \infty$ 时, $\widehat{\mu}_{r}\left(a_{k}\right) \rightarrow 0$.

定理 8.3 设 $\mu \geqslant 0,0<q<p \leqslant \infty$, 则下列各款互相等价:

(A) $T_{\mu}: F_{\varphi}^{p} \rightarrow F_{\varphi}^{q}$ 是有界算子;

(B) $T_{\mu}: F_{\varphi}^{p} \rightarrow F_{\varphi}^{q}$ 是紧算子;

(C) 存在 (或对任意) $t>0, \widetilde{\mu}_{t} \in L^{\frac{p q}{p-q}}$;

(D) 存在 (或对任意) $\delta>0, \widehat{\mu}_{\delta} \in L^{\frac{p q}{p-q}}$;

(E) 存在 (或对任意) $\mathbb{C}^{n}$ 上的 $r$ - 格 $\left.\left\{a_{k}\right\}_{k},\left\{\widehat{\mu}_{r}\left(a_{k}\right)\right)\right\}_{k} \in l^{\frac{p q}{p-q}}$. 进一步,

$$
\left.\left\|T_{\mu}\right\|_{F_{\varphi}^{p} \rightarrow F_{\varphi}^{q}} \simeq\left\|\widetilde{\mu}_{t}\right\|_{L^{\frac{p q}{p}-q}} \simeq\left\|\widehat{\mu}_{\delta}\right\|_{L^{\frac{p q}{p-q}}} \simeq \|\left\{\widehat{\mu}_{r}\left(a_{k}\right)\right)\right\}_{k} \|_{l^{\frac{p q}{p-q}}} .
$$

注 8.1 (1) 定理 8.1-8.3 摘自文献 [38,49]. 若 $1 \leqslant p=q<\infty$, 定理 8.1 和 8.2 便是文献 [24] 的 主要结果. 若 $p$ 或 $q$ 至少有一个取作 $\infty$, 定理 8.1-8.3 就是文献 [49] 的主要结论. 当 $1<p=q<\infty$, $\varphi(z)=\frac{\alpha}{2}|z|^{2}$ 时, 定理 8.1-8.3 就是文献 [10] 的主要结果. 进一步, 若 $p=q=2, \varphi$ 取作相应的特殊函 数时, 便能得到文献 $[6,17]$ 关于正 Toeplitz 算子有界性和紧性的刻画.

(2) 定理 8.1 和 8.2 表明, 当 $p \leqslant q$ 时, 正 Toeplitz 算子在 Fock 空间上的有界性 (或紧性) 与 $p$ 和 $q$ 甚至 $\frac{p}{q}$ 均无关, 即存在 $0<s \leqslant \infty$ 使得 $T_{\mu}$ 在 $F_{\varphi}^{s}$ 上是有界算子 (或紧算子) 当且仅当对任意 $0<p \leqslant q \leqslant \infty, T_{\mu}: F_{\varphi}^{p} \rightarrow F_{\varphi}^{q}$ 是有界算子 (或紧算子). 这与 Bergman 空间情形有很大差别.

(3) 给定 $\mathbb{C}$ 上满足 $\Delta \phi d v$ 为双倍测度的次调和函数 $\phi$, 我们也可讨论 Fock 空间 $F_{\phi}^{p}$ 上正 Toeplitz 算子的有界性和紧性特征. 由于此时的 Fock 空间更为一般 (如 (2.3) 不再成立), 所以, 相应的性质刻 画及定理证明较 $F_{\varphi}^{p}$ 的情形有着很大差别, 具体参见文献 [30].

Bergman 空间上正 Toeplitz 算子的 Schatten 类也已被深入研究, 如文献 [3,50-53] 等. 在 Fock 空 间情形, 2010 年, Isralowitz 和 Zhu ${ }^{[6]}$ 研究了正 Toeplitz 算子在经典 Fock 空间 $F_{\alpha}^{2}$ 上的 Schatten 类. 2015 年, Isralowitz 等人 ${ }^{[47]}$ 讨论了 $T_{\mu}$ 在 $F_{\varphi}^{2}$ 上的 Schatten 类性质, 得到如下定理.

定理 8.4 设 $\mu \geqslant 0,0<p \leqslant \infty, r>0$, 则下列各款互相等价:

(A) $T_{\mu} \in S_{p}\left(F_{\varphi}^{2}\right)$;

(B) $\widetilde{\mu} \in L^{p}$;

(C) $\widehat{\mu}_{r} \in L^{p}$;

(D) 存在 (或对任意) $\mathbb{C}^{n}$ 上的 $r$ - 格 $\left\{a_{k}\right\}_{k}$, 有 $\left\{\widehat{\mu}_{r}\left(a_{k}\right)\right\}_{k} \in l^{p}$.

注 8.2 定理 8.4 摘自文献 [47]. 取 $\varphi(z)=\frac{\alpha}{2}|z|^{2}, \alpha>0$, 该定理就是文献 [6] 的定理 4.4 和 5.4.

近年来, 有学者引入了 Schatten-Herz 类正 Toeplitz 算子. 2005 年, Loaiza 等人 ${ }^{[54]}$ 在单位圆盘上 首次提出了 Bergman 空间上 Schatten-Herz 类正 Toeplitz 算子 $S_{p, q}$, 并针对部分 $p, q$ 指标, 讨论了其 
特征刻画. 事实上, Schatten-Herz 类正 Toeplitz 算子是 Schatten 类正 Toeplitz 算子的推广. 随即, 有 关调和 Bergman 空间 (包括 $\mathbb{R}^{n}$ 中的单位球和上半平面) 上 Schatten-Herz 类正 Toeplitz 算子也吸引 了学者们的研究兴趣, 参见文献 $[50,55,56]$. 然而, 这些研究大部分都是在有界区域上进行的, 如单位 圆盘、单位球等, 即使在 $\mathbb{R}_{+}^{n+1}$ 这个无界区域, 研究也很大程度依赖于区域上可迁的 Möbius 变换群. 在文献 [57] 中, 我们给出了无界区域 $\mathbb{C}^{n}$ 上 Herz 空间和 $F_{\varphi}^{2}$ 上 Schatten-Herz 类正 Toeplitz 算子的定 义, 并讨论了 $T_{\mu}$ 属于 Schatten-Herz 类的充要条件. 在我们叙述结果前, 先给出相关定义.

给出复平面的分解: 令

$$
E_{k}=\left\{z \in \mathbb{C}^{n}: k-1 \leqslant|z|<k\right\}, \quad k=1,2, \ldots,
$$

则 $\mathbb{C}^{n}=\bigcup_{k=1}^{\infty} E_{k}$. 符号 $\chi_{k}$ 表示 $E_{k}$ 的特征函数.

定义 8.5 给定 $0<p, q \leqslant \infty$, Herz 空间 $\mathbf{K}_{q}^{p}$ 是指 $\mathbb{C}^{n}$ 上满足

$$
\|f\|_{\mathbf{K}_{q}^{p}}=\left\|\left\{\left\|f \chi_{k}\right\|_{L^{p}}\right\}_{k}\right\|_{l^{q}}<\infty
$$

的 Lebesgue 可测函数 $f$ 的全体. 进一步地, $\mathbf{K}_{0}^{p}$ 表示 $\mathbf{K}_{\infty}^{p}$ 中满足

$$
\lim _{k \rightarrow \infty}\left\|f \chi_{k}\right\|_{L^{p}}=0
$$

的 $f$ 的集合. 设 $\boldsymbol{a}=\left\{a_{m}\right\}_{m}$ 是 $\mathbb{C}^{n}$ 上的 $r$ - 格, $\left\{\xi_{m}\right\}_{m} \subseteq \mathbb{C}^{n}$. 记 $\left(\xi \chi_{k}\right)_{m}=\xi_{m} \chi_{k}\left(a_{m}\right), m=1,2, \ldots$ 给 定 $0<p, q \leqslant \infty$, 如果

$$
\left\|\left\{\xi_{m}\right\}_{m}\right\|_{l_{q}^{p}}=\left\{\sum_{k=1}^{\infty}\left(\sum_{a_{m} \in E_{k}}\left|\xi_{m}\right|^{p}\right)^{\frac{q}{p}}\right\}^{\frac{1}{q}}<\infty,
$$

则称 $\left\{\xi_{m}\right\}_{m}$ 属于离散 Herz 空间 $l_{q}^{p}(\boldsymbol{a})$. 进一步地, 若 $\left\{\xi_{m}\right\}_{m} \in l_{\infty}^{p}(\boldsymbol{a})$ 且

$$
\lim _{k \rightarrow \infty}\left\|\left\{\xi \chi_{k}\right\}_{m}\right\|_{l^{p}}=0
$$

则称 $\left\{\xi_{m}\right\}_{m} \in l_{0}^{p}(\boldsymbol{a})$.

Herz 空间 $\mathbf{K}_{q}^{p}$ 是经典 Lebesgue 空间的推广, $\mathbf{K}_{p}^{p}=L^{p}$. 有关 Herz 空间的更多研究可参见文献 [58]. 利用 Herz 空间, 我们可定义 Fock 空间 $F_{\varphi}^{2}$ 上 Schatten-Herz 类正 Toeplitz 算子, 参见文献 [59].

定义 8.6 设 $\mu \geqslant 0$, 符号 $\mu \chi_{k}$ 定义为 $\chi_{k} \mu(V)=\mu\left(V \cap E_{k}\right)$, 其中 $V \subset \mathbb{C}^{n}$ 为可测集. 给定 $0<p, q \leqslant \infty$, 如果

$$
\left\|T_{\mu}\right\|_{p, q}=\left\|\left\{\left\|T_{\mu \chi_{k}}\right\|_{S_{p}}\right\}_{k}\right\|_{l^{q}}<\infty,
$$

则称 $T_{\mu}$ 属于 $(p, q)$ Schatten-Herz 类, 记作 $T_{\mu} \in S_{p, q}\left(F_{\varphi}^{2}\right)$. 进一步地, 若 $T_{\mu} \in S_{p, \infty}\left(F_{\varphi}^{2}\right)$, 且当 $k \rightarrow \infty$ 时, 有 $\left\|T_{\mu \chi_{k}}\right\|_{S_{p}} \rightarrow 0$, 则称 $T_{\mu} \in S_{p, 0}\left(F_{\varphi}^{2}\right)$.

文献 [59] 讨论了 Fock 空间 $F_{\varphi}^{2}$ 上 Schatten-Herz 类正 Toeplitz 算子的特性, 即下面的定理 8.7. 比 较定理 8.4 和 8.7, 不难发现 Schatten-Herz 类正 Toeplitz 算子是 Schatten 类正 Toeplitz 算子的推广.

定理 8.7 设 $\mu \geqslant 0,0<p \leqslant \infty, 0 \leqslant q \leqslant \infty$, 则下列各款互相等价:
(A) $T_{\mu} \in S_{p, q}\left(F_{\varphi}^{2}\right)$;
(B) $\widetilde{\mu} \in \mathbf{K}_{q}^{p}$;
(C) 存在 (或对任意) $\delta>0, \widehat{\mu}_{\delta} \in \mathbf{K}_{q}^{p}$; 
(D) 存在 (或对任意) $\mathbb{C}^{n}$ 上的 $r$ - 格 $\boldsymbol{a}=\left\{a_{k}\right\}_{k},\left\{\widehat{\mu}_{r}\left(a_{k}\right)\right\}_{k} \in l_{q}^{p}(\boldsymbol{a})$. 进一步, 对 $0<p, q \leqslant \infty$, 成立

$$
\left\|T_{\mu}\right\|_{p, q} \simeq\|\widetilde{\mu}\|_{\mathbf{K}_{q}^{p}} \simeq\left\|\widehat{\mu}_{r}\right\|_{\mathbf{K}_{q}^{p}} \simeq\left\|\left\{\widehat{\mu}_{r}\left(a_{k}\right)\right\}_{k}\right\|_{l_{q}^{p}} .
$$

除了正 Toeplitz 算子, 以 BMO 函数为符号的 Toeplitz 算子也是人们的研究兴趣所在. 对 $g \in$ BMO, Coburn 等人 ${ }^{[15]}$ 讨论了 Toeplitz 算子 $T_{g}$ 在经典 Fock 空间 $F_{1}^{2}$ 上的有界性 (或紧性) 特征. 我们 将他们的结果推广到了 $0<p<\infty$, 参见文献 [38]. 在文献 [60], 对所有 $0<p<\infty$, 我们得到了 $\mathrm{BMO}$ 函数 $g$ 所诱导的算子 $T_{g}$ 在 Fock 空间 $F_{\varphi}^{p}$ 上的有界性和紧性特征. 我们先给出空间 BMO 的定义.

定义 8.8 设 $g$ 是 $\mathbb{C}^{n}$ 上的可测函数, 若对所有 $z \in \mathbb{C}^{n}, g(\cdot)\left|k_{\varphi, z}(\cdot)\right|^{2} \in L_{\varphi}^{1}$ 且

$$
\|g\|_{\mathrm{BMO}}=\sup _{z \in \mathbb{C}^{n}} \int_{\mathbb{C}^{n}}|g(w)-\widetilde{g}(z)|\left|k_{\varphi, z}(w)\right|^{2} \mathrm{e}^{-2 \varphi(w)} d A(w)<\infty,
$$

则称 $g$ 属于 BMO 空间.

定理 8.9 设 $0<p<\infty, g \in \mathrm{BMO}$, 则

(A) $T_{g}$ 在 $F_{\varphi}^{p}$ 上是有界算子的充要条件是 $\tilde{f}$ 在 $\mathbb{C}^{n}$ 上有界;

(B) $T_{g}$ 在 $F_{\varphi}^{p}$ 上是紧算子的充要条件是对任意 $r>0$, 成立

$$
\lim _{z \rightarrow \infty} \sup _{w \in B(z, r)}\left|\left\langle T_{f} k_{\varphi, z}, k_{\varphi, w}\right\rangle_{\varphi}\right|=0 .
$$

由文献 [61, 命题 1.5], 在经典 Fock 空间情形, (8.1) 等价于 $\lim _{z \rightarrow \infty} \widetilde{g}(z)=0$. 所以, 取 $\varphi(z)=\frac{1}{2}|z|^{2}$, 定理 8.9 就是文献 [38, 定理 4.3]. 进一步, 当 $p=2$ 时, 定理 8.9 便是文献 [15] 的主要结果.

\section{Hankel 算子}

除了 Toeplitz 算子, Hankel 算子也是特殊类算子研究中的重要对象. 有不少专著对 Hankel 算子进 行了详细讨论, 如文献 $[3,16,62,63]$. 直至今日, 仍有许多学者致力于这类算子的研究, 参见文献 [64-71] 等. 为了叙述的方便, 我们先介绍一些常用的空间以及 Fock 空间上 Hankel 算子的定义.

定义 9.1 设 $1 \leqslant p<\infty, L_{\mathrm{loc}}^{p}\left(\mathbb{C}^{n}\right)$ 表示 $\mathbb{C}^{n}$ 上局部 $p$ 次可积的 Lebesgue 可测函数的全体. 给定 $r>0$ 和 $f \in L_{\mathrm{loc}}^{p}\left(\mathbb{C}^{n}\right)$, 如果

$$
\|f\|_{\mathrm{BA}_{r}^{p}}=\sup _{z \in \mathbb{C}^{n}}\left(\frac{1}{v(B(z, r))} \int_{B(z, r)}|f|^{p} d v\right)^{\frac{1}{p}}<\infty,
$$

则称 $f \in \mathrm{BA}_{r}^{p}$. 空间 $\mathrm{VA}_{r}^{p}$ 表示 $\mathrm{BA}^{p}$ 中满足

$$
\lim _{z \rightarrow \infty} \frac{1}{v(B(z, r))} \int_{B(z, r)}|f|^{p} d v=0
$$

的函数 $f$ 的全体. 空间 $\mathrm{BMO}_{r}^{p}$ 表示 $L_{\mathrm{loc}}^{p}\left(\mathbb{C}^{n}\right)$ 中满足

$$
\|f\|_{\mathrm{BMO}_{r}^{p}}=\sup _{z \in \mathbb{C}^{n}} \mathrm{MO}_{p, r}(f)(z)<\infty
$$

的函数 $f$ 的全体, 其中

$$
\operatorname{MO}_{p, r}(f)(z)=\left(\frac{1}{v(B(z, r))} \int_{B(z, r)}\left|f-\widehat{f}_{r}(z)\right|^{p} d v\right)^{\frac{1}{p}} .
$$


如果 $f \in \mathrm{BMO}_{r}^{p}$ 且

$$
\lim _{z \rightarrow \infty} \mathrm{MO}_{p, r}(f)(z)=0
$$

则称 $f \in \mathrm{VMO}_{r}^{p}$.

由文献 [72] 可知, 空间 $\mathrm{BA}_{r}^{p}, \mathrm{VA}_{r}^{p}, \mathrm{BMO}_{r}^{p}$ 和 $\mathrm{VMO}_{r}^{p}$ 与 $r$ 无关, 所以可以分别简记为 $\mathrm{BA}^{p}, \mathrm{VA}^{p}$, $\mathrm{BMO}^{p}$ 和 $\mathrm{VMO}^{p}$.

定义 9.2 给定 $1 \leqslant p<\infty$ 和 $\mathbb{C}^{n}$ 上满足 $g k_{\varphi, z} \in L_{\varphi}^{p}$ 的可测函数 $g$ (简记为 $g \in \Gamma$ ), 由 $g$ 所诱导 的 Hankel 算子在 $F_{\varphi}^{p}$ 上 (稠) 定义为

$$
H_{g} f=g f-P(g f)=(I-P)(g f),
$$

其中 $I$ 表示 $L_{\varphi}^{p}$ 上的恒等算子, $P$ 为 Bergman 投影.

2005 年, Bauer ${ }^{[12]}$ 讨论了 Hankel 算子 $H_{g}$ 和 $H_{\bar{g}}$ 在经典 Fock 空间 $F_{1}^{2}$ 上同时为有界算子 (或紧 算子) 的充要条件. 2012 年, Zhu 的专著 [16] 讨论了 Hankel 算子 $H_{g}$ 在 $F_{\alpha}^{2}$ 上的相关特性, 包括有界 性、紧性和 Schatten 类. 最近, Perala 等人 ${ }^{[7]}$ 将有界性和紧性部分的结果推广到了 $1 \leqslant p<\infty$ 的情 形. 给定 $\mathbb{C}$ 上满足 $\Delta \phi d v$ 为双倍测度的次调和函数 $\phi$ 和整函数 $g$, Constantin 和 Ortega-Cerdà ${ }^{[29]}$ 研 究了 Hankel 算子 $H_{\bar{g}}$ 在 Fock 空间 $F_{\phi}^{2}$ 上分别为有界算子、紧算子和属于 Schatten 类的特征刻画. 给 定 $\mathbb{C}^{n}$ 上满足一定条件的另一类权函数 $\psi, 2013$ 年, Seip 和 Youssfi ${ }^{[33]}$ 获得了由全纯函数 $g$ 所诱导的 Hankel 算子 $H_{\bar{g}}$ 在加权 Fock 空间 $F_{\psi}^{2}$ 上的有界性、紧性和 Schatten 类特征. 2014 年, Wang 等人 ${ }^{[73]}$ 讨论了 $F_{\psi}^{2}$ 上 Hankel 算子 $H_{g}$ 和 $H_{\bar{g}}$ 同为有界算子 (或紧算子) 的充要条件. 对 $g \in \Gamma$, 我们在文献 [72] 研究了 Hankel 算子 $H_{g}$ 在 Fock 空间 $F_{\varphi}^{p}$ 上的有界性、紧性和 Schatten 类特征. 在列出文献 [72] 的结 果 (定理 9.3 和 9.4 ) 前, 需要引入 $\mathbb{C}^{n}$ 上函数 $G_{p}$,

$$
G_{p}(z)=\inf \left\{\left(\frac{1}{v(B(z, r))} \int_{B(z, r)}|g-h|^{p} d v\right)^{\frac{1}{p}}: h \in H(B(z, r))\right\}, \quad z \in \mathbb{C}^{n} .
$$

定理 9.3 设 $g \in \Gamma, 1 \leqslant p<\infty$, 则下列各款互相等价:

(A) $H_{g}: F_{\varphi}^{p} \rightarrow L_{\varphi}^{p}$ 是有界算子;

(B) 存在 $r \in(0,1]$ 使得函数 $G_{p}$ 在 $\mathbb{C}^{n}$ 上是有界的;

(C) $g$ 可分解为 $g=g_{1}+g_{2}$, 其中 $g_{1} \in \mathrm{BA}^{p}, g_{2} \in C^{1}\left(\mathbb{C}^{n}\right)$ 且 $\bar{\partial} g_{2}$ 在 $\mathbb{C}^{n}$ 上是有界的. 进一步,

$$
\left\|H_{g}\right\|_{F_{\varphi}^{p} \rightarrow L_{\varphi}^{p}} \simeq \sup _{z \in \mathbb{C}^{n}} G_{p}(z) \simeq \sup _{z \in \mathbb{C}^{n}}\left[\widehat{\left(\left|g_{1}\right|^{p}\right)_{r}}(z)\right]^{\frac{1}{p}}+\sup _{z \in \mathbb{C}^{n}}\left|\bar{\partial} g_{2}(z)\right| .
$$

定理 9.4 设 $g \in \Gamma, 1 \leqslant p<\infty$, 则下列各款互相等价:

(A) $H_{g}: F_{\varphi}^{p} \rightarrow L_{\varphi}^{p}$ 是紧算子;

(B) 存在 $r \in(0,1]$, 当 $z \rightarrow \infty$ 时, $G_{p}(z) \rightarrow 0$;

(C) $g$ 可分解为 $g=g_{1}+g_{2}$, 其中 $g_{1} \in \mathrm{VA}^{p}$ 且 $g_{2} \in C^{1}\left(\mathbb{C}^{n}\right)$ 满足 $\bar{\partial} g_{2}(z) \rightarrow 0(z \rightarrow \infty)$.

利用定理 9.3 和 9.4, 便能得到 $H_{g}$ 及 $H_{\bar{g}}$ 同时是 $F_{\varphi}^{p}$ 上有界算子 (或同时是紧算子) 的特征.

定理 9.5 设 $g \in \Gamma, 1 \leqslant p<\infty$, 则

(A) $F_{\varphi}^{p}$ 到 $L_{\varphi}^{p}$ 上的 $H_{g}$ 和 $H_{\bar{g}}$ 同为有界算子的充要条件是 $g \in \mathrm{BMO}^{p}$;

(B) $F_{\varphi}^{p}$ 到 $L_{\varphi}^{p}$ 上的 $H_{g}$ 和 $H_{\bar{g}}$ 同为紧算子的充要条件是 $g \in \mathrm{VMO}^{p}$.

如果 $g$ 是整函数, 利用定理 9.5 便能得到定理 9.6. 限制在 $n=1, p=2, \varphi(z)=\frac{\alpha}{2}|z|^{2}$ 时, 定理 9.6 就是文献 [16, 推论 8.6]. 
定理 9.6 设 $g \in \Gamma \cap H\left(\mathbb{C}^{n}\right), 1 \leqslant p<\infty$, 则在 $F_{\varphi}^{p}$ 上 $H_{g}=0$ 且

(A) $H_{\bar{g}}: F_{\varphi}^{p} \rightarrow L_{\varphi}^{p}$ 为有界算子的充要条件是 $\bar{\partial} g$ 在 $\mathbb{C}^{n}$ 上有界;

(B) $H_{\bar{g}}: F_{\varphi}^{p} \rightarrow L_{\varphi}^{p}$ 为紧算子的充要条件是当 $z \rightarrow \infty$ 时, $\bar{\partial} g(z) \rightarrow 0$.

在定理 9.5 和 9.6 中取 $\varphi(z)=\frac{1}{2}|z|^{2}$ 且 $p=2$, 就得文献 [12] 的定理 4.3 和 5.3; 限制在复平面上, 取 $\varphi(z)=\frac{\alpha}{2}|z|^{2}$, 就得到文献 [7] 的定理 2 和 4 ; 进一步, 当 $p=2$ 时, 定理 9.3 和 9.4 就推出文献 [16] 的定理 8.4 和 8.5 .

文献 [72] 关于单个 Hankel 算子 $H_{g}$ 有界性和紧性的处理方法也适用于文献 $[29,33]$ 所研究的 两类 Fock 空间. 也就是说, 在这两类 Fock 空间上可得到与定理 9.3-9.6 相平行的结果, 从而推广文 献 $[29,33]$.

Fock 空间上 Schatten 类 Hankel 算子的研究也已为人们所关注. 2004 年, 给定 $1 \leqslant p<\infty$, Xia 和 Zheng ${ }^{[74]}$ 在经典 Fock 空间 $F_{1}^{2}$ 上刻画了 Hankel 算子 $H_{g}$ 和 $H_{\bar{g}}$ 同时属于 Schatten- $p$ 类的充要条件 是 $\mathrm{MO}(g) \in L^{p}$, 其中 $\mathrm{MO}(g)(\cdot)=\sqrt{\widetilde{|g|^{2}}(\cdot)-|\widetilde{g}(\cdot)|^{2}} .2011$ 年, Isralowitz 将文献 [74] 的成果予以完善, 他在文献 [75] 中得到了 $0<p<1$ 时的相应结果. 在 $g$ 是整函数的前提下, 文献 $[16,29,33]$ 研究了 $H_{\bar{g}}$ 的 Schatten- $p$ 类性质. 我们在文献 [72] 讨论了单个 Hankel 算子 $H_{g}$ 的 Schatten 类, 不过 $p$ 的范围限 制在 $[2, \infty)$.

定理 9.7 设 $g \in \Gamma, 2 \leqslant p<\infty, H_{g}: F_{\varphi}^{2} \rightarrow L_{\varphi}^{2}$ 是有界的, 则下列各款互相等价:

(A) $H_{g} \in S_{p}$;

(B) 存在 (或对任意) $\mathbb{C}^{n}$ 上的 $\frac{r}{2}-$ 格 $\left\{a_{k}\right\}_{k}$, 有 $\left\{G_{2}\left(a_{k}\right)\right\}_{k} \in l^{p}$;

(C) $g$ 可分解为 $g=g_{1}+g_{2}$, 当 $h=g_{1}$ 或 $h=\bar{\partial} g_{2}$ 时满足

$$
\left[\widehat{\left(|h|^{2}\right)_{r}}(z)\right]^{\frac{1}{2}}=\left(\frac{1}{v(B(z, r))} \int_{B(z, r)}|h|^{2} d v\right)^{\frac{1}{2}} \in L^{p} .
$$

进一步,

$$
\left\|H_{g}\right\|_{S_{p}} \simeq\left\|\left\{G_{2}\left(a_{k}\right)\right\}_{k}\right\|_{l^{p}} \simeq\left\|\left[\widehat{\left(\left|g_{1}\right|^{2}\right)_{r}}(z)\right]^{\frac{1}{2}}\right\|_{L^{p}}+\left\|\bar{\partial} g_{2}\right\|_{L^{p}} .
$$

注 9.1 (1) 类似地, 对文献 $[29,33]$ 所研究的这两类 Fock 空间, 也可得到相应的定理. 进一步, 取 $g$ 是整函数, 就能得到文献 $[29$, 定理 5$]$ 和 [33, 定理 F].

(2) 在经典 Fock 空间上, 利用 $\mathbb{C}^{n}$ 的双全纯自同构所诱导的 Weyl 变换 (参见文献 [16, 第 76 页]) 便可得到 $0<p<\infty$ 时 Schatten 类 Hankel 算子的完整刻画. 而对 $\varphi(z)=\frac{\alpha}{2}|z|^{2}$ 以外的权函数所诱导 的 Fock 空间而言, 现有结果只是对 $p \geqslant 2$ 而得, 要得到完整的结果还需有方法上的创新.

\section{0 弱局部 Toeplitz 算子代数}

Toeplitz 算子代数是 $C^{*}$ - 代数的一个特殊种类, 已被广泛研究. 在 Bergman 空间情形, 设 $T$ 是 Toeplitz 算子乘积 $T_{g_{1}} \cdots T_{g_{m}}$ 的有限和, 其中 $g_{k}$ 均为单位圆盘上的有界函数, 1998 年, Axler 和 Zheng ${ }^{[76]}$ 证明了 $T$ 为紧算子当且仅当其 Berezin 变换 $\widetilde{T}(z)$ 满足条件 $\lim _{|z| \rightarrow 1} \widetilde{T}(z)=0$. Engliš ${ }^{[77]}$ 将 这一结果推广至 $\mathbb{C}^{n}$ 有界对称域上的 Bergman 空间. 在经典 Fock 空间上, 2013 年, Xia 和 Zheng ${ }^{[78]}$ 在 $F_{\alpha}^{2}$ 上定义了 “强局部算子 (strongly localized operators)”, 证明了由强局部算子所生成的闭代数是 一个 $C^{*}$ - 代数, 还证明了该闭代数中的元素 $T$ 是紧算子的充要条件是 $\widetilde{T}$ 在无穷点处的极限为 0 . 根 据文献 [78, 命题 4.1], 有界函数所诱导的 Toeplitz 算子必定是强局部算子. 因此, 文献 [76] 的结论 
在经典 Fock 空间上也成立并被进一步一般化. Isralowitz 将文献 [78] 的结果推广到了 Fock 空间 $F_{\varphi}^{2}$ 上, 参见文献 [61]. 限定于 $1<p<\infty$, Isralowitz 等人在 $F_{\varphi}^{p}$ 上定义了 “弱局部算子 (weakly localized operators)”, 这是比强局部算子更为一般的一类算子. 文献 [79] 证明了由 “弱局部算子” 所生成的闭 代数是一个 $C^{*}$ - 代数, 并获证该闭代数中的元素 $T$ 是 $F_{\varphi}^{p}$ 上紧算子的充要条件是 $\lim _{z \rightarrow \infty} \widetilde{T}(z)=0$. 2014 年, 我们将 $F_{\varphi}^{p}$ 上 “弱局部算子” 的定义扩充到了 $0<p \leqslant 1$ 情形. 记 $\mathcal{D}=\operatorname{span}\left\{k_{\varphi, z}: z \in \mathbb{C}^{n}\right\}$ 为 规范化 Bergman 核 $k_{\varphi, z}$ 所张成的线性空间, 则对所有 $0<p \leqslant \infty, \mathcal{D}$ 在 $F_{\varphi}^{p}$ 中稠密. 这里, 我们总认为 线性算子 $T$ 的定义域包含 $\mathcal{D}$, 且 $T K_{\varphi, z}$ 关于 $z$ 是共轭全纯的, 其中 $K_{\varphi, z}(\cdot)=K_{\varphi}(\cdot, z)$. 下面关于弱局 部算子的定义摘自文献 $[60,79]$.

定义 10.1 设 $0<p<\infty, T: \mathcal{D} \rightarrow F_{\varphi}^{\infty}$ 是线性算子. 如果成立

$$
\begin{aligned}
& \sup _{z \in \mathbb{C}^{n}} \int_{\mathbb{C}^{n}}\left|\left\langle T k_{\varphi, z}, k_{\varphi, w}\right\rangle_{\varphi}\right|^{s} d A(w)<\infty, \\
& \sup _{z \in \mathbb{C}^{n}} \int_{\mathbb{C}^{n}}\left|\left\langle k_{\varphi, z}, T k_{\varphi, w}\right\rangle_{\varphi}\right|^{s} d A(w)<\infty, \\
& \lim _{r \rightarrow \infty} \sup _{z \in \mathbb{C}^{n}} \int_{B(z, r)^{c}}\left|\left\langle T k_{\varphi, z}, k_{\varphi, w}\right\rangle_{\varphi}\right|^{s} d A(w)=0, \\
& \lim _{r \rightarrow \infty} \sup _{z \in \mathbb{C}^{n}} \int_{B(z, r)^{c}}\left|\left\langle k_{\varphi, z}, T k_{\varphi, w}\right\rangle_{\varphi}\right|^{s} d A(w)=0,
\end{aligned}
$$

其中 $s=\min \{1, p\}$, 则称 $T$ 是 $F_{\varphi}^{p}$ 上的弱局部算子. 用 $\mathrm{WL}_{\varphi}^{p}$ 表示 $F_{\varphi}^{p}$ 上的弱局部算子所生成的代数. 用 $\overline{\mathrm{WL}}_{\varphi}^{p}$ 表示 $\mathrm{WL}_{\varphi}^{p}$ 在 $F_{\varphi}^{p}$ 算子范数下的闭包. 当 $\varphi(z)=\frac{\alpha}{2}|z|$ 时, $\mathrm{WL}_{\varphi}^{p}$ 和 $\overline{\mathrm{WL}}_{\varphi}^{p}$ 分别简记为 $\mathrm{WL}_{\alpha}^{p}$ 和 $\overline{\mathrm{WL}}_{\alpha}^{p}$.

首先给出 $\mathrm{WL}_{\varphi}^{p}$ 的两个主要性质: 定理 10.2 和 10.3. 当 $0<p \leqslant 1$ 时, 这两个定理可见于文献 [60]; 当 $1<p<\infty$ 时, 它们可见于文献 [79].

定理 10.2 设 $0<p<\infty$. 若 $T \in \mathrm{WL}_{\varphi}^{p}$, 则 $T$ 是 $F_{\varphi}^{p}$ 上的有界算子.

定理 10.3 当 $0<p \leqslant 1$ 时, $\mathrm{WL}_{\varphi}^{p}$ 是 $C^{*}$ - 代数; 当 $1<p<\infty$ 时, $\overline{\mathrm{WL}}_{\varphi}^{p}$ 是 $C^{*}$ - 代数.

在 $1<p<\infty$ 情形, 我们还不知道 $\mathrm{WL}_{\varphi}^{p}$ 是否也是闭的.

下述定理是关于 $\overline{\mathrm{WL}}_{\varphi}^{p}$ 中元素成为 $F_{\varphi}^{p}$ 上的紧算子的特征刻画.

定理 10.4 设 $0<p<\infty, T \in \overline{\mathrm{WL}}_{\varphi}^{p}$, 则下列各款互相等价:

(A) $T$ 是 $F_{\varphi}^{p}$ 上的紧算子;

(B) 对任意 $r>0, \lim _{z \rightarrow \infty} \sup _{w \in B(z, r)}\left|\left\langle T k_{\varphi, z}, k_{\varphi, w}\right\rangle_{\varphi}\right|=0$;

(C) $\lim _{z \rightarrow \infty} \sup _{w \in \mathbb{C}^{n}}\left|\left\langle T k_{\varphi, z}, k_{\varphi, w}\right\rangle_{\varphi}\right|=0$;

(D) $\lim _{z \rightarrow \infty}\left\|T k_{\varphi, z}\right\|_{p, \varphi}=0$.

定理 10.4 见于文献 [60], 它推广了文献 [79] 的主要结果. 根据文献 [61, 命题 1.5], 在经典 Fock 空 间情形, 条件 (B) 等价于 $\lim _{z \rightarrow \infty} \widetilde{g}(z)=0$. 所以, 取 $p=2$ 和 $\varphi(z)=\frac{1}{2}|z|^{2}$, 由定理 10.4 便能得到文 献 [78] 的主要结论.

在 Bergman 空间情形, 2007 年由 Suarez 证明了 $A^{2}$ 上的紧算子就是由有界函数诱导的 Toeplitz 算子所生成的闭代数中满足其 Berezin 变换趋于 0 的那些算子, 参见文献 [80]. Mitkovski 等人 ${ }^{[81]}$ 将 这一结果推广到加权 Bergman 空间上. 对 $0<p<\infty$, 以 $\mathcal{T}_{\varphi}^{p}$ 表示有界函数所诱导的 Toeplitz 算子在 $F_{\varphi}^{p}$ 算子范数下生成的闭代数. 当 $\varphi(z)=\frac{\alpha}{2}|z|^{2}$ 时, $\mathcal{T}_{\varphi}^{p}$ 简记为 $\mathcal{T}_{\alpha}^{p}$. 由文献 $[60,79]$ 可知, $\mathcal{T}_{\varphi}^{p} \subseteq \overline{\mathrm{WL}}_{\varphi}^{p}$. 2012 年, Bauer 和 Isralowitz ${ }^{[11]}$ 获得了经典 Fock 空间 $F_{\alpha}^{p}$ 上的紧算子与 $\mathcal{T}_{\alpha}^{p}$ 中的元素之间的关系, 即 定理 10.5. 
定理 10.5 设 $1<p<\infty, T$ 是 $F_{\alpha}^{p}$ 上的有界算子, 则 $T$ 为紧算子的充要条件是 $T \in \mathcal{T}_{\alpha}^{p}$ 且 $\lim _{z \rightarrow \infty} \widetilde{T}(z)=0$, 其中 $\widetilde{T}(z)=\left\langle T k_{\alpha, z}, k_{\alpha, z}\right\rangle_{\alpha}$.

给定满足 $d d^{c} \varphi \simeq \omega_{0}$ 的权函数 $\varphi$, Isralowitz ${ }^{[61]}$ 研究了 $F_{\varphi}^{p}$ 上紧算子与有界函数所诱导的 Toeplitz 算子代数的关系, 获得了如下结果.

定理 10.6 设 $1<p<\infty, T$ 是 $F_{\varphi}^{p}$ 上的有界算子, 则 $T$ 为紧算子的充要条件是 $T \in \mathcal{T}_{\varphi}^{p}$ 且对任 意 $r>0$, 有

$$
\lim _{z \rightarrow \infty} \sup _{w \in B(z, r)}\left|\left\langle T k_{\varphi, z}, k_{\varphi, w}\right\rangle_{\varphi}\right|=0 .
$$

当 $\varphi(z)=\frac{\alpha}{2}|z|^{2}$ 时, 由定理 10.6 就能得到定理 10.5.

当 $0<p \leqslant 1$ 时, 定理 10.6 不再成立. 事实上, 存在 $F_{\varphi}^{p}$ 上的紧算子 $T$, 但 $T \notin \mathcal{T}_{\varphi}^{p}$. 例如, 由文 献 $\left[16\right.$, 引理 4.39] 可知, 存在 $\mathbb{C}$ 的分离序列 $\left\{z_{j}\right\}_{j=1}^{\infty}$ 和 $\mathbb{C}$ 上的函数 $f \in F_{\alpha}^{\infty}$, 使得

$$
f\left(z_{j}\right) \mathrm{e}^{-\frac{\alpha}{2}\left|z_{j}\right|^{2}}=1, \quad j=1,2, \ldots
$$

对 $0<p \leqslant 1$, 取定 $\mathbb{C}$ 上的函数 $h \in F_{\alpha}^{p}$ 满足 $h(0) \neq 0$. 定义 $F_{\alpha}^{p}$ 上的算子 $T$ 为

$$
T(\cdot)=\langle\cdot, g\rangle_{\alpha} h
$$

显然, $T$ 是秩为 1 的有界算子, 故 $T$ 是 $F_{\alpha}^{p}$ 上的紧算子. 但 $T \notin \mathrm{WL}_{\alpha}^{p}=\overline{\mathrm{WL}}_{\alpha}^{p}$, 从而 $T \notin \mathcal{T}_{\alpha}^{p}$. 相对于定 理 10.4 的条件 (D), 这个例子还说明, 在 $0<p \leqslant 1$ 时, 存在 $F_{\varphi}^{p}$ 上的紧算子使得

$$
\lim _{z \rightarrow \infty}\left\|T k_{\alpha, z}\right\|_{p, \alpha} \neq 0
$$

具体可参见文献 $[60]$.

比 $\mathcal{T}_{\varphi}^{p}$ 更为一般的是由满足 $\widetilde{|g|}$ 有界的 $g$ 诱导的 Toeplitz 算子所生成的闭代数. 记 $\mathcal{B}_{p}^{\varphi}$ 表示 Toeplitz 算子有限积 $T_{f_{1}} T_{f_{2}} \cdots T_{f_{m}}$ 的线性组合在 $F_{\varphi}^{p}$ 算子范数下的闭包, 其中 $f_{j} \in$ BMO 且 $\widetilde{f}_{j}$ 有界.

定理 10.7 设 $0<p<\infty, S \in \mathcal{B}_{p}^{\varphi}$, 则 $S$ 是 $F_{\varphi}^{p}$ 上的紧算子当且仅当对任意 $r>0$, 有

$$
\lim _{z \rightarrow \infty} \sup _{w \in B(z, r)}\left|\left\langle S k_{\varphi, z}, k_{\varphi, w}\right\rangle_{\varphi}\right|=0 .
$$

定理 10.7 见于文献 [60]. 限制在 $\varphi(z)=\frac{1}{2}|z|^{2}$ 及 $p=2$, 它由 Isralowitz [14] 首先获得. 定理 10.7 同时涵盖了文献 $[15,38]$ 的相应结果.

各类函数空间上有界算子的本性模范数也是函数空间与算子理论的研究内容之一. Bergman 空间 上复合算子、广义 Cesàro 算子、Toeplitz 算子等的本性模范数研究可以参见文献 [80-84] 等. 设 $T$ 是 $F_{\varphi}^{p}$ 上的有界算子, $T$ 的本性模范数定义为

$$
\|T\|_{e, F_{\varphi}^{p}}=\inf \left\{\|T-A\|_{F_{\varphi}^{p} \rightarrow F_{\varphi}^{p}}: A \text { 是 } F_{\varphi}^{p} \text { 上的紧算子 }\right\} .
$$

2014 年, 给定 $1<p<\infty$ 和 $T \in \overline{\mathrm{WL}}_{\varphi}^{p}$, Isralowitz 等人 ${ }^{[79]}$ 获得了 $\|T\|_{e, F_{\varphi}^{p}}$ 的上界估计. 在 $0<p \leqslant 1$ 的前提下, 我们给出了 $\|T\|_{e, F_{\varphi}^{p}}$ 的等价估计, 参见文献 [60].

定理 10.8 设 $T \in \overline{\mathrm{WL}}_{\varphi}^{p}$, 则

(A) 当 $0<p \leqslant 1$ 时,

$$
\|T\|_{e, F_{\varphi}^{p}} \simeq \limsup _{z \rightarrow \infty}\left\|T k_{\varphi, z}\right\|_{p, \varphi}
$$


(B) 当 $1<p<\infty$ 时,

$$
\|T\|_{e, F_{\varphi}^{p}} \leqslant C \limsup _{z \rightarrow \infty}\left\|T k_{\varphi, z}\right\|_{p, \varphi} .
$$

当 $1<p<\infty$ 时, 定理 10.8 只给出了 $T$ 的上界估计, 自然可以问类似的下界估计是否成立? 或 是考虑更弱的问题: 给定 $1<p<\infty$ 和有界函数 $g$, 关于 Toeplitz 算子 $T_{g}$ 是否成立

$$
\left\|T_{g}\right\|_{e, F_{\varphi}^{p}} \simeq \limsup _{z \rightarrow \infty}\left\|T_{g} k_{\varphi, z}\right\|_{p, \varphi} ?
$$

\section{1 后记}

本文所用的 Fock 空间这一名称, 在当今学界其实还没有统一的称呼. 除了 Fock 空间之外, 它还 被称作 Bargmann 空间或 Segal-Bargmann 空间, 等等. 而我们采用 Fock 空间这个术语仅仅是因为自 身的习惯, 再有就是所见的文献大部分也是采用了这样的称谓.

全纯函数理论与调和函数理论有着紧密联系, 两者交叉融合、互相促进. 类似于复变量的 Fock 空 间, 显然可以平行地定义 $\mathbb{R}^{n}$ 上的调和函数所组成的实变量 Fock 空间. 但较之于复变量 Fock 空间, 迄今为止, 人们对实变量 Fock 空间的认识还十分有限. 2010 年, Engliš [85] 给出了实变量 Fock 空间 的定义并利用超几何函数 (hypergeometric function) 得到了该空间的 Bergman 核函数表示, 并讨论了 Berezin 变换的相关特性. 而对实 Fock 空间及相关算子的后继研究几乎还是空白. 对调和 Fock 空间 上 Toeplitz 算子和 Hankel 算子等的研究还需要有对 Bergman 核函数的增长估计以及由 Bergman 核 所诱导的度量的研究为前提.

最后, 我们需要说明此综述报告只是选取了 Fock 空间及相关算子研究中我们感兴趣的那部分内 容, 文献的选取也只局限于我们的视野范围之内.

\section{参考文献}

1 Duren P L. Theory of $H^{p}$ Spaces. Amsterdam: Academic Press, 1970

2 Hedenmalm H, Korenblum B, Zhu K H. Theory of Bergman Spaces. GTM 199. New York: Springer, 2000

3 Zhu K H. Operator Theory in Function Spaces. Mathematical Surveys and Monographs, vol. 138. Providence: Amer Math Soc, 2007

4 Bargmann V. On a Hilbert space of analytic functions and an associated integral transform. Comm Pure Appl Math, 1961, 14: 187-214

5 Furdui O. On a class of integral operators. Integral Equations Operator Theory, 2010, 66: 469-483

6 Isralowitz J, Zhu K H. Toeplitz operators on the Fock space. Integral Equations Operator Theory, 2010, 66: 593-611

7 Perälä A, Schuster A, Virtanen J A. Hankel operators on Fock spaces. Oper Theory Adv Appl, 2014, 236: 377-390

8 Dostanić M, Zhu K H. Integral operators induced by the Fock kernel. Integral Equations Operator Theory, 2008, 60: 217-236

9 Bauer W, Coburn L A, Isralowitz J. Heat flow, BMO, and the compactness of Toeplitz operators. J Funct Anal, 2010, 259: $57-78$

10 Hu Z J, Lv X F. Toeplitz operators from one Fock space to another. Integral Equations Operator Theory, 2011, 70: $541-559$

11 Bauer W, Isralowitz J. Compactness characterization of operators in the Toeplitz algebra of the Fock space $F_{\alpha}^{p}$. J Funct Anal, 2012, 263: 1323-1355

12 Bauer W. Mean oscillation and Hankel operators on the Segal-Bargmann space. Integral Equations Operator Theory, 2005, 52: 1-15

13 Gryc W E, Kemp T. Duality in Segal-Bargmann spaces. J Funct Anal, 2011, 261: 1591-1623

14 Isralowitz J. Compact Toeplitz operators on the Segal-Bargmann space. J Math Anal Appl, 2011, 374: 554-557 
15 Coburn L A, Isralowitz J, Li B. Toeplitz operators with BMO symbols on the Segal-Bargmann space. Trans Amer Math Soc, 2011, 363: 3015-3030

16 Zhu K H. Analysis on Fock Spaces. New York: Springer, 2012

17 Cho H R, Zhu K H. Fock-Sobolev spaces and their Carleson measures. J Funct Anal, 2012, 263: 2483-2506

18 Cho H R, Choe B R, Koo H. Fock-Sobolev spaces of fractional order. Potential Anal, 2015, 43: 199-240

19 Cho H R, Choe B R, Koo H. Combinations of composition operators on the Fock-Sobolev spaces. Potential Anal, 2014, 41: 1223-1246

20 Choe B R, Yang J. Commutants of Toeplitz operators with radial symbols on the Fock-Sobolev space. J Math Anal Appl, 2014, 415: 779-790

21 Mengestie T. Carleson type measures for Fock-Sobolev spaces. Complex Anal Oper Theory, 2014, 8: 1225-1256

22 Wang X F, Cao G F, Xia J. Toeplitz operators on Fock-Sobolev spaces with positive measure symbols. Sci China Math, 2014, 57: 1443-1462

23 Wang X F, Xia J, Cao G F. Bounded, compact and $S_{p}$-class operators on Fock-Sobolev spaces (in Chinese). Sci Sin Math, 2014, 44: 263-274

24 Schuster A P, Varolin D. Toeplitz operators and Carleson measures on generalized Bargmann-Fock spaces. Integral Equations Operator Theory, 2012, 72: 363-392

25 Christ M. On the $\bar{\partial}$ equation in weighted $L^{2}$ norms in $\mathbb{C}$. J Geom Anal, 1991, 1: 193-230

26 Delin H. Pointwise estimates for the weighted Bergman projection kernel in $\mathbb{C}^{n}$, using a weighted $L^{2}$ estimate for the $\bar{\partial}$ equation. Ann Inst Fourier (Grenoble), 1998, 48: 967-997

27 Marco N, Massaneda X, Ortega-Cerdà J. Interpolating and sampling sequences for entire functions. Geom Funct Anal, 2003, 13: 862-914

28 Marzo J, Ortega-Cerdà J. Pointwise estimates for the Bergman kernel of the weighted Fock space. J Geom Anal, 2009, 19: $890-910$

29 Constantin O, Ortega-Cerdà J. Some spectral properties of the canonical solution operator to $\bar{\partial}$ on weighted Fock spaces. J Math Anal Appl, 2011, 377: 353-361

$30 \mathrm{Hu}$ Z J, Lv X F. Positive Toeplitz operators between different weighted Fock spaces. Submitted

31 Schneider G, Schneider K. Generalized Hankel operators on the Fock space. Math Nachr, 2009, 282: 1811-1826

32 Schneider G, Schneider K. Generalized Hankel operators on the Fock space II. Math Nachr, 2011, 284: 1967-1984

33 Seip K, Youssfi E H. Hankel operators on Fock spaces and related Bergman kernel estimates. J Geom Anal, 2013, 23: 170-201

34 Constantin O, Peláez J. Integral operators, embedding theorems and a Littlewood-Paley formula on weighted Fock spaces. J Geom Anal, doi: 10.1007/s12220-015-9585-7, 2015

$35 \mathrm{Hu}$ Z J. Extended Cesaro operators on mixed norm spaces. Proc Amer Math Soc, 2003, 131: 2171-2179

$36 \mathrm{Hu}$ Z J. Equivalent norms on Fock spaces with some application to extended Cesaro operators. Proc Amer Math Soc, 2013, 141: 2829-2840

37 Constantin O. A Volterra-type integration operator on Fock spaces. Proc Amer Math Soc, 2012, 140: 4247-4257

38 Hu Z J, Lv X F. Toeplitz operators on Fock spaces $F^{p}(\varphi)$. Integral Equations Operator Theory, 2014, 80: 33-59

39 Zhu K. Positive Toeplitz operators on weighted Bergman spaces of bounded symmetric domains. J Operator Theory, 1988, 20: 329-357

40 Luecking D H. Embedding theorems for spaces of analytic functions via Khinchine's inequality. Michigan Math J, 1993, 40: 333-358

41 Choe B R, Lee Y J, Na K. Positive Toeplitz operators from a harmonic Bergman space into another. Tohoku Math J, 2004, 56: $255-270$

42 Carswell B, MacCluer B D, Schuster A. Composition operators on the Fock spaces. Acta Sci Math (Szeged), 2003, 74: $807-827$

43 Choe B R, Izuchi K, Koo H. Linear sums of two composition operators on the Fock space. J Math Anal Appl, 2010, 369: $112-119$

44 Zygmund A. Trigonometric Series. 3rd ed. Combridge: Combridge University Press, 2002

45 Mengestie T. Volterra type and weighted composition operators on weighted Fock spaces. Integral Equations Operator Theory, 2013, 76: 81-94

46 Mengestie T. Product of Volterra type integral and composition operators on weighted Fock spaces. J Geom Anal, 2014, 24: 740-755

47 Isralowitz J, Virtanen J, Wolf L. Schatten class Toeplitz operators on generalized Fock spaces. J Math Anal Appl, 2015, 421: 329-337 
48 Mengestie T. On Toeplitz operators between Fock spaces. Integral Equations Operator Theory, 2014, 78: 213-224

49 Lu J, Lv X F. Toeplitz operators between Fock spaces. Bull Aust Math Soc, 2015, 92: 316-324

50 Choe B R, Koo H, Lee Y. Positive Schatten class Toeplitz operators on the ball. Studia Math, 2008, 189: 65-90

51 Hu Z J, Tang X M. Schatten(-Herz) class extended Cesàro operators on Bergman spaces in the unit ball of $\mathbb{C}^{n}$. Proc Amer Math Soc, 2010, 138: 2803-2814

52 Zhu K H. Schatten class Toeplitz operators on weighted Bergman spaces of the unit ball. New York J Math, 2007, 13: 299-316

53 Pau J. A remark on Schatten class Toeplitz operators on Bergman spaces. Proc Amer Math Soc, 2014, 142: 2763-2768

54 Loaiza M, López-García M, Pérez-Esteva S. Herz classes and Toeplitz operators in the disk. Integral Equations Operator Theory, 2005, 53: 287-296

55 Choe B R, Koo H, Na K. Positive Toeplitz operators of Schatten-Herz type. Nagoya Math J, 2007, 185: 31-62

56 Choe B R, Nam K. Toeplitz operators and Herz spaces on the half-space. Integral Equations Operator Theory, 2007, 59: $501-521$

57 Lv X F, Hu Z J. Schatten-Herz class Toeplitz operators on harmonic Bergman spaces of smooth domains in $R^{n}$. Math Nachr, 2013, 286: 248-259

58 Herenández E, Yang D. Interpolation of Herz spaces and applications. Math Nachr, 1999, 205: 69-87

59 Lv X F, Hu Z J. Schatten-Herz classes of Toeplitz operators on the generalized Fock space. Submitted

$60 \mathrm{Hu}$ Z J, Lv X F, Wick B. Weakly localized operators on Fock spaces with application to compactness. Submitted

61 Isralowitz J. Compactness and essential norm problems of operators on generalized Fock spaces. ArXiv:1305.7475, 2013

62 Partington J R. An Introduction to Hankel Operators. London Mathematical Society Student Texts, 13. Cambridge: Cambridge University Press, 1988

63 Peller V V. Hankel Operators and Their Applications. Springer Monographs in Mathematics. New York: SpringerVerlag, 2003

64 Stroethoff K. Hankel and Toeplitz operators on the Fock space. Michigan Math J, 1992, 39: 3-16

65 Schneider G. Hankel operators with antiholomorphic symbols on the Fock space. Proc Amer Math Soc, 2004, 132: 2399-2409

66 Bauer W. Hilbert-Schmidt Hankel operators on the Segal-Bargmann space. Proc Amer Math Soc, 2004, 132: 2989-2996

67 Knirsch W, Schneider G. Generalized Hankel operators and the generalized solution operator to $\bar{\partial}$ on the Fock space and on the Bergman space of the unit disc. Math Nachr, 2006, 279: 1684-1694

68 Knirsch W, Schneider G. Continuity and Schatten-von Neumann p-class membership of Hankel operators with antiholomorphic symbols on (generalized) Fock spaces. J Math Anal Appl, 2006, 320: 403-414

69 Bommier-Hato H, Youssfi E H. Hankel operators on weighted Fock spaces. Integral Equations Operator Theory, 2007, 59: $1-17$

70 Fang Q L, Xia J B. A local inequality for Hankel operators on the sphere and its application. J Funct Anal, 2014, 266: 876-930

71 Le T. Hilbert-Schmidt Hankel operators over complete Reinhardt domains. Integral Equations Operator Theory, 2014, 78: $515-522$

72 Hu Z J, Lv X F. Hankel operators on weighted Fock spaces (in Chinese). Sci Sin Math, doi: 10.1360/012015-19, 2016

73 Wang X F, Cao G F, Zhu K H. BMO and Hankel operators on Fock-type spaces. J Geom Anal, 2014, 25: 1650-1665

74 Xia J B, Zheng D C. Standard deviation and Schatten class Hankel operators on the Segal-Bargmann space. Indiana Univ Math J, 2004, 53: 1381-1399

75 Isralowitz J. Schatten $p$ class Hankel operators on the Segal-Bargmann space $H^{2}\left(\mathbb{C}^{n}, d \mu\right)$ for $0<p<1$. J Operator Theory, 2011, 66: 145-160

76 Axler S, Zheng D. Compact operators via Berezin transforms. Indiana Math J, 1998, 47: 387-400

77 Englis M. Compact Toeplitz operators via the Berezin transform on bounded symmetric domains. Integral Equations Operator Theory, 1999, 33: 426-455

78 Xia J B, Zheng D C. Localization and Berezin transform on the Fock space. J Funct Anal, 2013, 264: 97-117

79 Isralowitz J, Mitkovski M, Wick B. Localization and compactness in Bergman and Fock spaces. ArXiv:1306.0316, 2014

80 Suarez D. The essential norm of operators in the Toeplitz algebra of $A^{p}\left(\mathbf{B}_{n}\right)$. Indiana Univ Math J, 2007, 56: 2186-2232

81 Mitkovski M, Suarez D, Wick B D. The essential norm of operators on $A_{\alpha}^{p}\left(B_{n}\right)$. Integral Equations Operator Theory, 2013, 75: 197-233

82 Hu Z J. Essential norm of extended Cesàro operators from one Bergman space to another. Bull Aust Math Soc, 2012, 85: $307-314$ 
83 Galindo P, Laitila J, Lindström M. Essential norm estimates for composition operators on BMOA. J Funct Anal, 2013, 265: 629-643

84 Laitila J, Lindström M. The essential norm of a weighted composition operator on BMOA. Math Z, 2015, 279: 423-434

85 Engliš M. Berezin transform on the harmonic Fock space. J Math Anal Appl, 2010, 367: 75-97

\title{
Fock spaces and relative operators
}

\section{HU ZhangJian \& LV XiaoFen}

\begin{abstract}
This paper is a timely survey on generalized Fock spaces and operators acting on them. It contains the definition of Fock spaces with various weights, the precise estimates on the Bergman kernel, the equivalent norms and the Carleson measures. Also, some recent progress on the study of certain operators on Fock spaces is introduced, which includes composition operators, extended Cesàro operators, Toeplitz operators, Hankel operators, and those in the closed algebra generated by weakly localized operators.
\end{abstract}

Keywords Fock space, Bergman kernel, Carleson measure, Toeplitz operator, Hankel operator $\operatorname{MSC}(2010) \quad 47 \mathrm{~B} 38$

doi: $10.1360 /$ N012015-00141 